\title{
Do Patients with Prostate Cancer Benefit from Exercise Interventions? A Systematic Review and Meta-Analysis
}

\author{
Martin Færch Andersen $1, * \mathbb{D}$, Julie Midtgaard ${ }^{2,3}$ and Eik Dybboe Bjerre 4 \\ 1 Department of Physiotherapy, University College of Northern Denmark, Selma Lagerløfs Vej 2, \\ DK-9200 Aalborg, Denmark \\ 2 Mental Health Centre Glostrup, University of Copenhagen, Nordstjernevej 41, DK-2600 Glostrup, Denmark; \\ julie.midtgaard.klausen@regionh.dk \\ 3 Department of Clinical Medicine, University of Copenhagen, Blegdamsvej 3B, DK-2200 Copenhagen, Denmark \\ 4 The University Hospitals' Centre for Health Research, Rigshospitalet, Ryesgade 27, DK-2200 Copenhagen, Denmark; \\ Eik_bjerre@hotmail.com \\ * Correspondence: mfan@ucn.dk
}

Citation: Andersen, M.F.; Midtgaard, J.; Bjerre, E.D. Do Patients with Prostate Cancer Benefit from Exercise Interventions? A Systematic Review and Meta-Analysis. Int. J. Environ. Res. Public Health 2022, 19, 972. https://doi.org/10.3390/ ijerph19020972

Academic Editor: Paul B. Tchounwou

Received: 30 November 2021

Accepted: 12 January 2022

Published: 15 January 2022

Publisher's Note: MDPI stays neutral with regard to jurisdictional claims in published maps and institutional affiliations.

Copyright: (c) 2022 by the authors. Licensee MDPI, Basel, Switzerland. This article is an open access article distributed under the terms and conditions of the Creative Commons Attribution (CC BY) license (https:// creativecommons.org/licenses/by/ $4.0 /)$.

\begin{abstract}
Men diagnosed and treated for prostate cancer experience severe adverse effects on quality of life (QoL) and metabolic health, some of which may be preventable or reversible with exercise, the benefits of which healthcare providers and patients increasingly acknowledge, though existing evidence on its effects varies in significance and magnitude. We aimed to review the effect of exercise on QoL and metabolic health in a broad prostate cancer population. A systematic search was conducted in nine databases and eligible trials were included in the meta-analytic procedure. All outcomes were stratified into aerobic exercise, resistance exercise, and a combination of both. The review identified 33 randomised controlled trials (2567 participants) eligible for inclusion. Exercise had a borderline small positive effect on cancer-specific QoL (standardised mean difference (SMD) $=0.10$, $95 \%$ confidence interval (CI) $-0.01-0.22)$, and a moderate to large effect on cardiovascular fitness $(\mathrm{SMD}=0.46,95 \% \mathrm{CI} 0.34-0.59)$ with aerobic exercise being the superior modality $(\mathrm{SMD}=0.60,95 \%$ CI 0.29-0.90). A positive significant effect was seen in lower body strength, whole-body fat mass, general mental health, and blood pressure. No significant effect was seen in fatigue, lean body mass, and general physical health. We thereby conclude that exercise is effective in improving metabolic health in men diagnosed with prostate cancer, with aerobic exercise as the superior modality. The effect of exercise on QoL was small and not mediated by choice of exercise modality.
\end{abstract}

Keywords: quality of life; metabolic health; prostate cancer; exercise; frailty; older adults; health

\section{Introduction}

According to the Global Cancer Observatory's GLOBOCAN 2018 database [1], prostate cancer is the most frequent cancer type affecting men in Western nations, with an estimated 1.2 million incident cases annually worldwide. Progress in screening and treatment has resulted in increased survival rates, though not without severe adverse effects on patients' physical and mental health, such as the increased risk of metabolic disease and reduced quality of life (QoL) [2,3]. Many of these adverse effects are treatment related. The disease stage and its risk of metastasis, combined with the patient's overall condition, determine treatment options. When the disease is localised, patients are offered curative treatment, including surgery or radiotherapy. However, when the disease is metastatic at the time of diagnosis, patients are offered androgen-deprivation therapy (ADT), androgen receptor signalling inhibitors (ARSI) and chemotherapy, with palliative intent. It is estimated that almost $50 \%$ of patients will receive ADT or ARSI at one point during their treatment [4]. These men, especially those treated with ARSI, are more likely to develop metabolic conditions, such as type 2 diabetes, cardiovascular disease, abdominal obesity, or bone loss, potentially leading to osteopenia, osteoporosis, and bone fractures [5]. Furthermore, 
ADT is associated with a decline in QoL and sexual function, as well as the aggravation of fatigue [2]. Lastly, patients receiving ADT have a higher risk of other-cause mortality [6].

Some of these adverse effects may be preventable or reversible with exercise $[7,8]$, which is increasingly recognised by healthcare providers and patients. Moreover, exercise has been found to improve patient-reported outcomes (PRO) such as QoL and fatigue $[7,9,10]$ but also physiological outcomes such as cardiovascular fitness, muscle strength, and endurance $[7,8,11,12]$ in men diagnosed with prostate cancer. Additionally, general physical activity has been associated with reduced overall and prostate cancerspecific mortality [13].

While various reviews investigating the effect of exercise on patients diagnosed with prostate cancer [7-12] have been published previously, these have focused exclusively on either PROs [10], cardiovascular health for patients on ADT [11], or the effect of resistance exercise specifically [12]. Only one comprehensive systematic review with a meta-analysis addressing the effect of exercise in men diagnosed with prostate cancer has been published previously. However, this was conducted in 2015 [7] and since then, the number of trials evaluating exercise interventions for prostate cancer patients has doubled. Therefore, we aimed to offer an updated systematic review examining the potential differential effect of specific exercise modalities in the broad population of prostate cancer patients, to support clinicians' decision making when prescribing exercise.

The objective was to review and synthesise the effects of exercise interventions on QoL and metabolic health in men diagnosed with prostate cancer, and to examine possible differential effects across specific exercise modalities.

\section{Materials and Methods}

\subsection{Protocol and Registration}

This systematic review was conducted according to the Preferred Reporting Items for Systematic reviews and Meta-Analyses (PRISMA) (Supplementary Material File S6). A protocol was registered on the International Prospective Register of Systematic Reviews, number CRD42020153851.

\subsection{Eligibility Criteria}

We included all randomised controlled trials (RCTs), allowing randomisation on both the individual and cluster level and feasibility trials. The following three criteria had to be met: (1) Participants with a history of clinically diagnosed prostate cancer regardless of tumour histology or tumour stage, and that had received cancer treatment. We did not include studies with a mixed cancer population (breast, lung etc); (2) Exercise, defined as " ... a subset of physical activity that is planned, structured, and repetitive and has as a final or an intermediate objective the improvement or maintenance of physical fitness" [14], lasting at least eight weeks with components of aerobic and/or resistance exercise and reported details in relation to frequency, intensity, and duration. We defined aerobic exercise as any form of physical activity that produces an increased heart rate and respiratory volume to meet the oxygen requirements of the activated muscles [15]. Resistance exercise was defined as exercise that intends to improve or maintain the ability to create the most significant amount of power at a specific or prescheduled speed and type of muscle contraction [16]. Studies were included regardless of whether interventions were supervised or home-based and regardless of delivery method (individual, group, or mixed). Combinations with other types of interventions (dietary, psycho/social) were allowed; (3) Comparison groups receiving usual care or waiting-list protocols were included. We allowed comparisons with attention control groups, such as stretching protocols, but only objective outcomes were examined in these cases.

\subsection{Search Strategy}

A systematic search was conducted in June 2021. We identified literature from the following electronic databases: MEDLINE, EMBASE, Cochrane Central Register of Controlled 
Trials (CENTRAL), CINAHL, PsycINFO, SPORTDiscus, PEDroand Scopus ${ }^{\circledR}$. Supplementary Material File S1 contains the complete MEDLINE search strategy. We also expanded the database search by scanning reference lists of included studies or relevant reviews identified through the systematic search. Possible relevant unpublished studies were searched for in the grey literature via the OpenGrey database. Lastly, we screened ClinicalTrials.gov (https:/ / clinicaltrials.gov/, accessed on 11 August 2020) for ongoing trials.

\subsection{Study Selection}

Two authors (M.F.A. and E.D.B.) independently applied a three-step process to screen for eligibility: (1) screening of titles and abstracts to remove irrelevant studies; (2) fulltext screening of potentially relevant studies to assess compliance with eligibility criteria; and (3) linking of multiple publications from the same trial by comparing author names, location, specific details, number of participants, date, and duration. A lack of consensus at all stages was resolved by discussion with a third author (J.M.).

\subsection{Data Collection Process}

Data were extracted on the following outcomes: cancer-specific QoL, cardiovascular fitness, cancer-related fatigue, general physical and mental health, lower body strength, lean body mass, whole-body fat mass, and blood pressure. M.F.A. extracted and entered data on an Excel (Microsoft Corporation, Redmond, WA, USA) spreadsheet that E.D.B. then independently checked for accuracy. If more than one publication was identified for each trial, we selected the first published publication as the primary reference.

\subsection{Data Items}

For all outcomes, if more than two time points (i.e., baseline and post-intervention) were reported, we selected what was closest to the end of the intervention for extraction.

\subsection{Risk of Bias in Individual Studies}

Risk of bias in individual studies was assessed using Cochrane's risk of bias tool [17]. We excluded two domains, "blinding of the participants and healthcare providers" and "blinding of the outcome assessor of subjective outcomes" (self-reported), which were not relevant due to the nature of the intervention and because the participants were the assessors.

\subsection{Summary of Measurements}

Outcomes were presented as continuous values and effect estimates of individual trials calculated as either weighted mean difference (WMD) or standardised mean difference (SMD) with a 95\% confidence interval (CI). WMD was used when an outcome was assessed with an identical instrument in all included trials, while SMD was used in cases with different assessment instruments measuring the same outcome between trials [18]. For trials presenting multiple intervention arms, all relevant interventions contributed to the metaanalysis. Each arm had a control group allocated by dividing the number of participants in the original control group into two equal groups, creating single pair-wise comparisons [18]. Post-intervention measurement and post-intervention standard deviation (SD) were used in the meta-analysis. If post-intervention SD was not reported, either baseline SD or a calculated SD from reported standard error (SE) was used.

\subsection{Synthesis of Results}

The meta-analytic procedure was conducted following a two-stage process. First, a summary statistic was calculated to describe the observed effect of the intervention in each trial. Second, a pooled effect estimate was calculated for each outcome using a random effect model which involves an assumption that the effect being estimated in different trials are not identical but follows a distribution. 
Heterogeneity was tested using the chi-squared test, placing the significance level at 0.10 [18]. The quantity of heterogeneity was tested using the I-squared test [18]. Interpretation of the I-squared values was: $25 \%$ indicating low heterogeneity, $50 \%$ moderate heterogeneity, and 75\% considerable heterogeneity [19].

\subsection{Risk of Bias across Studies}

To account for the possibility of publication bias a funnel plot was conducted for visual examination of asymmetry and statistically tested using Egger's test [20], but these were only performed if a sufficient number of studies $(>10)$ were available [21].

\subsection{Additional Analyses}

To explore potential heterogeneity, prespecified subgroup analyses were conducted, which involves stratifying the studies according to intervention modality, i.e., all outcomes were stratified into aerobic exercise, resistance exercise and a combination of the two. Furthermore, outcomes were stratified according to type of assessment instrument.

The subsequent sensitivity analyses were conducted to test the robustness of the results: change score values, random versus fixed effect, excluding cluster-randomised studies, excluding pilot/feasibility studies and excluding outliers. All analyses were performed using STATA version 15.1. (StataCorp LLC, College Station, TX, USA)

\section{Results}

\subsection{Study Selection}

A systematic literature search was conducted in August 2020. Of the 6693 identified records, 33 trials (2567 participants) were found to be eligible for inclusion in this systematic review [22-54]. We identified 24 linked publications with follow-up measurements or secondary analyses [55-77]. Figure 1 illustrates the study selection process.

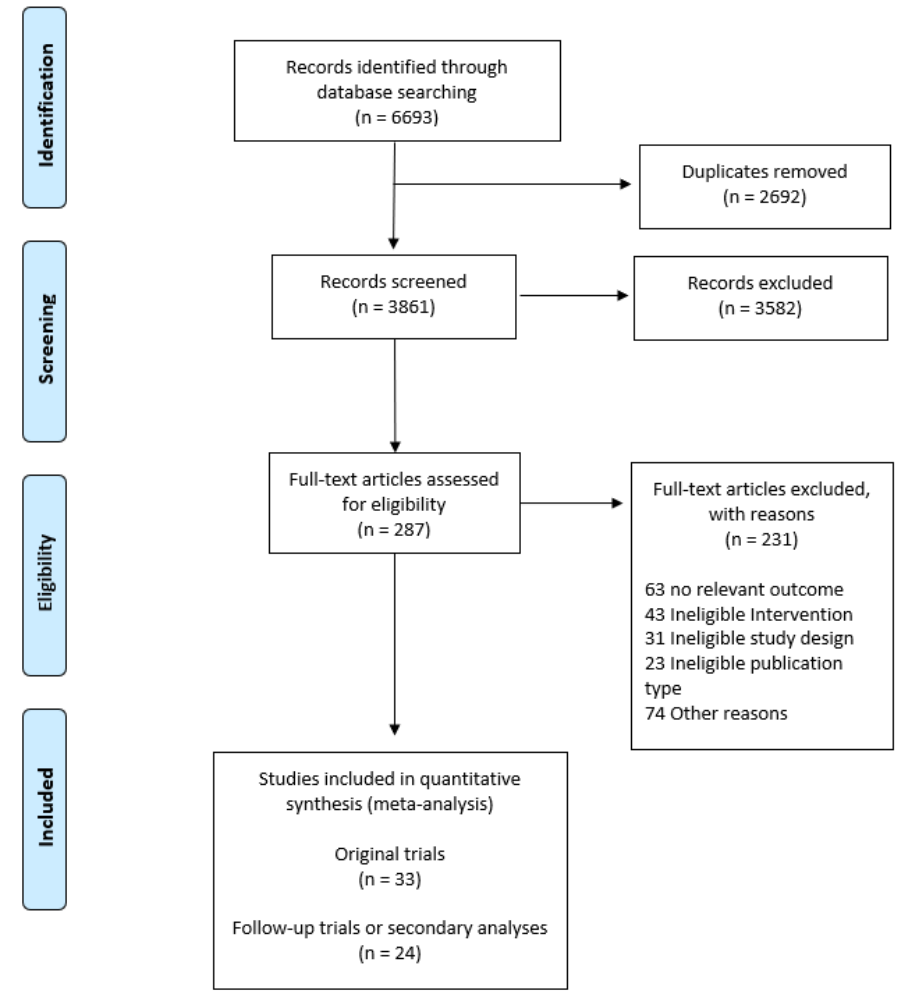

Figure 1. Flow diagram of literature and study selection process according to the 2009 Preferred Reporting Items of Systematic reviews and Meta-Analyses (PRISMA) with modification. Follow-up trials or secondary analyses represent reports involving the same study population for the same intervention trial. 


\subsection{Study Characteristics}

Table 1 provides an alphabetical list of detailed within-study characteristics. While only RCTs were included in this systematic review, there was one cluster-randomised trial [39] and six randomised feasibility trials or pilot studies $[24,25,27,29,30,35]$. Patients included received different treatment modalities across and within the 33 included trials. Fifteen trials reported primarily patients on ADT treatment $[20,22-25,27,30,38,40,42,44-46,48,49]$, six trials reported a combination of ADT and radiotherapy $[28,29,32,33,39,43]$ and one trial reported radiotherapy alone [37]. Others reported previous prostatectomy [35,41], and previous surgery, radiotherapy or a combination of those [31]. Two trials reported patients undergoing active surveillance $[26,34]$. Three trials reported patients receiving various treatments such as radiotherapy, surgery, hormone therapy [51]; surgery, radiotherapy, ADT or a combination [36]; and watchful waiting, ADT or castration [19]. One trial did not report information on treatment [21].

Two trials compared two exercise intervention groups that met the eligibility criteria [31,47]. The patients with prostate cancer in the included trials varied both within and across trials regarding treatment modalities, tumour stage and aggressiveness. Likewise, the exercise interventions (e.g., frequency, duration, exercise modalities and combinations with other therapeutic components) varied across the trials: Bourke et al. [23] combined aerobic exercise and resistance exercise with dietary and behavioural therapy: Bourke et al. [24] combined aerobic exercise with behavioural therapy; Dieperink et al. [28] combined resistance exercise with pelvic floor exercises and nurse consulting; Eriksen et al. [29] and O'Neill et al. [43] combined aerobic exercise with dietary advice; Focht et al. [30] combined aerobic exercise and resistance exercise with dietary and cognitive therapy; and Hebert et al. [34] combined aerobic exercise with dietary and stress reduction therapy. One exercise arm in Taaffe et al. [47,48] and in Winters-Stone et al. [52] had a combination of resistance exercise and impact training, such as landing, jumping and skipping. Park et al. [44] combined resistance exercise with pelvic floor exercises. Mardani et al. [54] combined aerobic and resistance exercise with flexibility and pelvic floor exercise. Two studies, Uth et al. [49] and Bjerre et al. [22], investigated football interventions. Most trials used usual care or waiting-list comparator, while four trials used an attention control group. Assessment instruments for measuring outcome of interest varied across trials. For the outcome cancer-specific QoL, trials used either a prostate cancer-specific patient-reported outcome measure (PROM) (e.g., Functional Assessment of Cancer Therapy-Prostate) [22,23,27,38,40,41,43,45,46] or general cancer PROM (e.g., European Organization for Research and Treatment of Cancer Quality of Life QuestionnaireC30) $[24,31,35,36,39,42,54]$. For the outcome cardiovascular fitness, trials used either a maximum testing procedure (i.e., VO2peak/max) $[23,24,29,37,38,40,41,46,49,51]$ or a submaximal walking test (i.e., $400 \mathrm{~m}$ walk test or 6 min walk test) $[25-27,30-33,35,36,39,42,43,47,50]$. 
Table 1. Study characteristics in alphabetic order.

\begin{tabular}{|c|c|c|c|c|c|c|c|}
\hline $\begin{array}{l}\text { Author } \\
\text { (Year) }\end{array}$ & $\begin{array}{c}\text { Sample } \\
\text { (Group } \\
\text { Randomised) }\end{array}$ & Population & $\begin{array}{l}\text { Intervention } \\
\text { Duration } \\
\text { (Follow-Up) }\end{array}$ & $\begin{array}{l}\text { Intervention } \\
\text { Frequency }\end{array}$ & Intervention Description & Control Group & Outcomes of Interest \\
\hline $\begin{array}{l}\text { Bjerre et al., } \\
\text { (2018) [22] }\end{array}$ & $\begin{array}{c}121 \\
(109 / 105)\end{array}$ & $\begin{array}{c}\text { Tumour stage: Gleason } \\
\text { mean 2.0-10.0 } \\
\text { Metastatic disease: } 19 \% \\
\text { PCa treatment: } \\
\text { No treatment, ADT, } \\
\text { castration }\end{array}$ & 24 weeks & 2 times per week & $\begin{array}{l}\text { Supervised } \\
\text { AT: } 20 \text { min of warm-up, } 20 \mathrm{~min} \text { of } \\
\text { dribbling and shooting, } 20 \mathrm{~min} \text { of } \\
\text { 5-7-a-side football }\end{array}$ & Usual care & $\begin{array}{l}\text { QoL }^{\mathrm{a}} \\
\text { Lean body mass }(\mathrm{kg})^{\mathrm{b}} \\
\text { Body fat mass }(\mathrm{kg})^{\mathrm{b}} \\
\text { General physical and } \\
\text { mental health }{ }^{\mathrm{c}}\end{array}$ \\
\hline $\begin{array}{l}\text { Bourke et al., } \\
\text { (2014) [23] }\end{array}$ & $\begin{array}{c}100 \\
(50 / 50)\end{array}$ & $\begin{array}{c}\text { Tumour stage: T } 3-4 \\
\text { Metastatic disease: } \\
20 \% \\
\text { PCa treatment: ADT }\end{array}$ & 12 weeks & $\begin{array}{l}\text { First } 6 \text { weeks: } 2 \\
\text { supervised, } 1 \text { home } \\
\text { Last } 6 \text { weeks: } 1 \\
\text { supervised, } 2 \text { home }\end{array}$ & $\begin{array}{c}\text { Supervised } \\
\text { AE: } 30 \text { min } 55-75 \% \text { max heartrate or } \\
\text { Borg } 11-13 \\
\text { RT: } 2-4 \text { sets } 8-12 \text { reps, } 60 \% 1 \mathrm{RM} \\
\text { progressed through intervention } \\
\text { Home } \\
\text { AE + RT: } 30 \text { min of skills taught in } \\
\text { supervised sessions }\end{array}$ & Usual care & $\begin{array}{c}\text { QoL }^{\mathrm{a}} \\
\text { Fatigue }^{\mathrm{d}} \\
\text { Cardiovascular fitness }^{\mathrm{e}} \\
\text { Systolic and diastolic BP }\end{array}$ \\
\hline $\begin{array}{l}\text { Bourke et al., } \\
\text { (2018) [24] }\end{array}$ & $\begin{array}{c}50 \\
(25 / 25)\end{array}$ & $\begin{array}{l}\text { Tumour stage: T 1-2 } \\
\text { Metastatic disease: } \\
\text { None } \\
\text { PCa treatment: No } \\
\text { information }\end{array}$ & 12 weeks & 2 times per week & $\begin{array}{c}\text { Supervised } \\
\text { AE: } 20-30,65-85 \% \text { of age-specific } \\
\text { heart rate max } \\
\text { Home } \\
\text { AE: } 150 \text { min moderate } \\
\text { cardiovascular fitness per week }\end{array}$ & Usual care & $\begin{array}{l}\mathrm{QoL}^{\mathrm{f}} \\
\text { Systolic and diastolic BP } \\
\text { Cardiovascular fitness }{ }^{\text {e }}\end{array}$ \\
\hline $\begin{array}{c}\text { Cormie et al., } \\
\text { (2013) [25] }\end{array}$ & $\begin{array}{c}20 \\
(10 / 10)\end{array}$ & $\begin{array}{c}\text { Tumour stage: Gleason } \\
\text { mean } 8.2 \\
\text { Metastatic disease: All } \\
\text { (inclusion criteria) } \\
\text { PCa treatment: Previous } \\
\text { ADT }\end{array}$ & 12 weeks & 2 times per week & $\begin{array}{c}\text { Supervised } \\
\text { RT: 1-4 set (NI reps), 12-6 RM, } 8 \\
\text { exercises } \\
\text { Home } \\
\text { AE: } 150 \text { min of moderate } \\
\text { cardiovascular fitness per week }\end{array}$ & $\begin{array}{l}\text { Waiting-list } \\
\text { protocol }\end{array}$ & $\begin{array}{c}\text { General physical and } \\
\text { mental health } \\
\text { Fatigue }^{\mathrm{h}} \\
\text { Cardiovascular fitness }^{\mathrm{i}} \\
\text { Lower body strength }^{\mathrm{j}} \\
{\text { Lean body mass }(\mathrm{kg})^{\mathrm{b}}}^{\mathrm{b}} \\
{\text { Body fat mass }(\mathrm{kg})^{\mathrm{b}}}^{\mathrm{b}}\end{array}$ \\
\hline $\begin{array}{l}\text { Cormie et al., } \\
\text { (2015) [26] }\end{array}$ & $\begin{array}{c}63 \\
(32 / 31)\end{array}$ & $\begin{array}{c}\text { Tumour stage: Gleason } \\
\text { mean 7.5 } \\
\text { Metastatic disease: } \\
\text { None (excluded) } \\
\text { PCa treatment: ADT }\end{array}$ & 12 weeks & 2 times per week & $\begin{array}{c}\text { Supervised } \\
\text { RT: } 1-4 \text { set (NI reps), 12-6 RM, } 8 \\
\text { exercises } \\
\text { AE: } 20-30 \text { min } 70-85 \% \text { of max heart } \\
\text { rate } \\
\text { Home } \\
\text { AE: } 150 \text { min of moderate } \\
\text { cardiovascular fitness per week }\end{array}$ & $\begin{array}{l}\text { Waiting-list } \\
\text { protocol }\end{array}$ & $\begin{array}{l}\text { General physical and } \\
\text { mental health } \mathrm{g} \\
\text { Fatigue }^{\mathrm{k}} \\
\text { Cardiovascular fitness }^{\mathrm{i}} \\
\text { Lower body strength }^{\mathrm{k}} \\
{\text { Lean body mass }(\mathrm{kg})^{\mathrm{b}}}^{\mathrm{b}} \\
\text { Body fat mass }(\mathrm{kg})^{\mathrm{b}} \\
\text { Systolic and diastolic BP }\end{array}$ \\
\hline
\end{tabular}


Table 1. Cont.

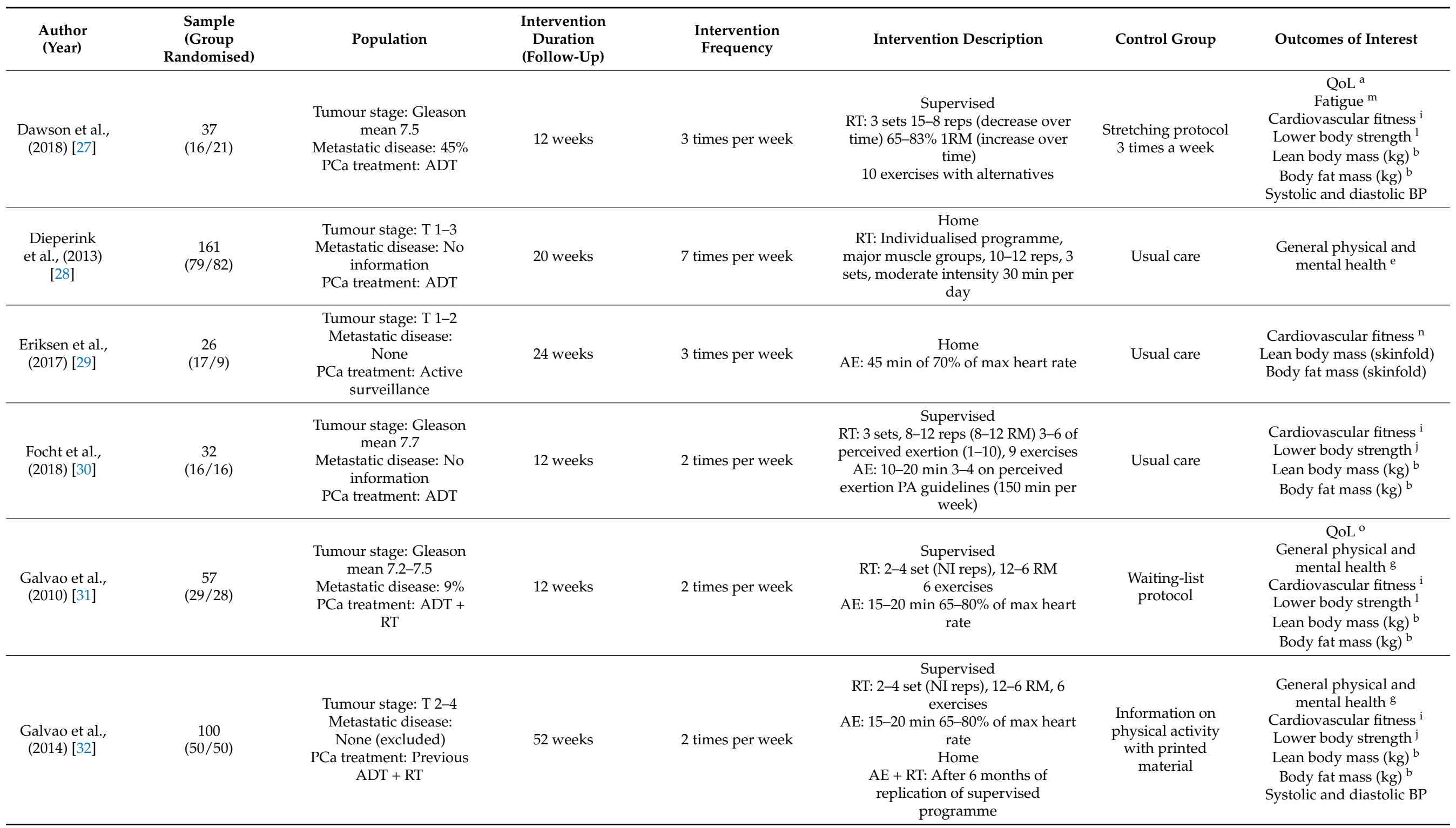


Table 1. Cont.

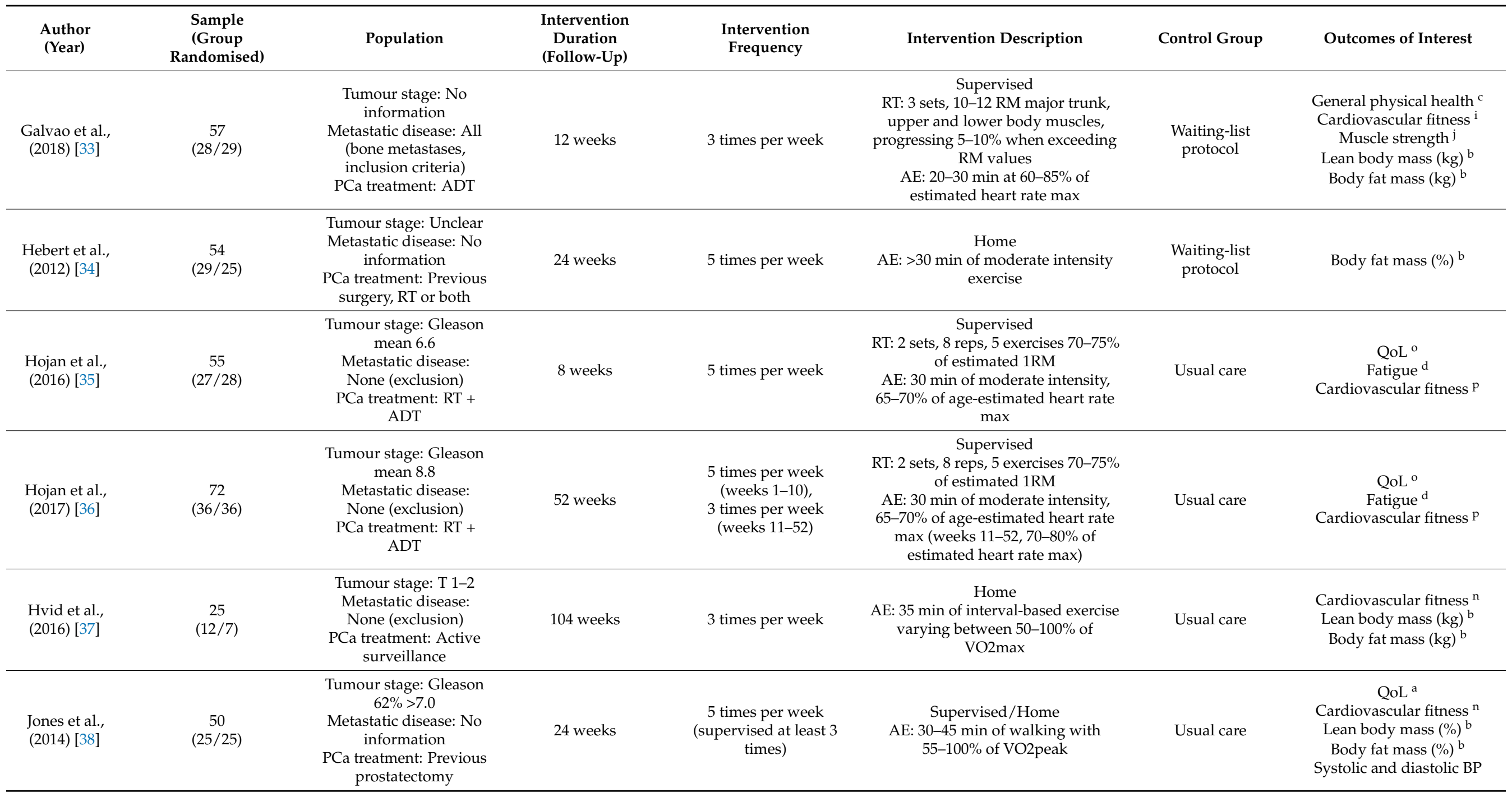


Table 1. Cont.

\begin{tabular}{|c|c|c|c|c|c|c|c|}
\hline $\begin{array}{l}\text { Author } \\
\text { (Year) }\end{array}$ & $\begin{array}{c}\text { Sample } \\
\text { (Group } \\
\text { Randomised) }\end{array}$ & Population & $\begin{array}{l}\text { Intervention } \\
\text { Duration } \\
\text { (Follow-Up) }\end{array}$ & $\begin{array}{l}\text { Intervention } \\
\text { Frequency }\end{array}$ & Intervention Description & Control Group & Outcomes of Interest \\
\hline $\begin{array}{l}\text { Livingston } \\
\text { et al., (2015) } \\
\text { [39] }\end{array}$ & $\begin{array}{c}147 \\
(54 / 92)\end{array}$ & $\begin{array}{l}\text { Tumour stage: T 1-3 } \\
\text { Metastatic disease: No } \\
\text { information } \\
\text { PCa treatment: Surgery, } \\
\text { RT, ADT or combined }\end{array}$ & 12 weeks & $\begin{array}{c}3 \text { times per week (2 } \\
\text { supervised, } 1 \text { home) }\end{array}$ & $\begin{array}{c}\text { Supervised } \\
\text { RT: 4-6 exercises, } 2 \text { sets, } 8-12 \text { reps } \\
\text { AE: } 20-30 \text { min } 40-70 \% \text { of max heart } \\
\text { rate } \\
\text { Home } \\
\text { RT + AE With bodyweight and } \\
\text { resistance band }\end{array}$ & Usual care & $\begin{array}{l}\text { QoL }^{\circ} \\
\text { Cardiovascular fitness }^{\mathrm{p}} \\
\text { Lower body Strength }^{1} \\
\text { Systolic and diastolic BP }\end{array}$ \\
\hline $\begin{array}{l}\text { Monga et al., } \\
(2007)[40]\end{array}$ & $\begin{array}{l}(30) 21 \\
(11 / 10)\end{array}$ & $\begin{array}{c}\text { Tumour stage: Gleason } \\
\text { mean } 5.3 \\
\text { Metastatic disease: } \\
\text { Unclear } \\
\text { PCa treatment: RT } \\
\end{array}$ & 8 weeks & 3 times per week & $\begin{array}{c}\text { Supervised } \\
\text { AE: } 30 \text { min walking on treadmill, } \\
65 \% \text { of heart rate reserve }\end{array}$ & Usual care & $\begin{array}{c}\text { QoL }^{\mathrm{a}} \\
\text { Fatigue }^{\mathrm{r}} \\
\text { Lower body strength }^{\mathrm{s}} \\
\text { Cardiovascular fitness }^{\mathrm{e}}\end{array}$ \\
\hline $\begin{array}{l}\text { Nilsen et al., } \\
\text { (2015) [42] }\end{array}$ & $\begin{array}{c}58 \\
(28 / 30)\end{array}$ & $\begin{array}{c}\text { Tumour stage: } \\
\text { Intermediate high-risk } \\
\text { profile } \\
\text { Metastatic disease: } \\
\text { Unclear } \\
\text { PCa treatment: RT + } \\
\text { ADT }\end{array}$ & 18 weeks & 3 times per week & $\begin{array}{c}\text { Supervised } \\
\text { RT: } 9 \text { exercises, Monday: } 1-3 \text { sets, } 10 \\
\text { RM, Fridays: } 2-3 \text { sets, } 6 \text { RM, } \\
\text { Wednesdays: submaximal session, } \\
10 \text { reps, } 80-90 \% \text { of } 10 \text { RM, } 2-3 \text { sets }\end{array}$ & Usual care & $\begin{array}{c}\text { OoL }^{\mathrm{o}} \\
\text { Fatigue }^{\mathrm{t}} \\
\text { Cardiovascular fitness }^{\mathrm{u}} \\
{\text { Lean body mass }(\mathrm{kg})^{\mathrm{b}}}^{\mathrm{b}} \\
{\text { Body fat mass }(\mathrm{kg})^{\mathrm{b}}} \\
\text { Lower body strength }^{1}\end{array}$ \\
\hline
\end{tabular}


Table 1. Cont.

\begin{tabular}{|c|c|c|c|c|c|c|c|}
\hline $\begin{array}{l}\text { Author } \\
\text { (Year) }\end{array}$ & $\begin{array}{l}\text { Sample } \\
\text { (Group } \\
\text { Randomised) }\end{array}$ & Population & $\begin{array}{l}\text { Intervention } \\
\text { Duration } \\
\text { (Follow-Up) }\end{array}$ & $\begin{array}{l}\text { Intervention } \\
\text { Frequency }\end{array}$ & Intervention Description & Control Group & Outcomes of Interest \\
\hline $\begin{array}{l}\text { O'Neill et al., } \\
\text { (2015) [43] }\end{array}$ & $\begin{array}{c}94 \\
(47 / 47)\end{array}$ & $\begin{array}{c}\text { Tumour stage: Gleason } \\
90 \% \geq 7.0 \\
\text { Metastatic disease: } \\
\text { Unclear } \\
\text { PCa treatment: ADT }\end{array}$ & 24 weeks & 5 times per week & $\begin{array}{c}\text { Home } \\
\text { AE: } 30 \text { min of brisk walking } \\
\text { (moderate intensity) }\end{array}$ & $\begin{array}{l}\text { Waiting-list } \\
\text { protocol }\end{array}$ & $\begin{array}{c}\text { QoL }^{\mathrm{a}} \\
\text { Fatigue }^{\mathrm{v}} \\
{\text { Lean body mass }(\mathrm{kg})^{\mathrm{b}}}^{\mathrm{b}} \\
{\text { Body fat mass }(\mathrm{kg})^{\mathrm{b}}}^{\mathrm{Cardiovascular} \mathrm{fitness}} \mathrm{p}\end{array}$ \\
\hline $\begin{array}{l}\text { Park et al., } \\
\text { (2012) [44] }\end{array}$ & $\begin{array}{c}66 \\
(33 / 33)\end{array}$ & $\begin{array}{l}\text { Tumour stage: T 2-3 } \\
\text { Metastatic disease: } \\
\text { Unclear } \\
\text { PCa treatment: Radical } \\
\text { prostatectomy }\end{array}$ & 12 weeks & 2 times per week & $\begin{array}{c}\text { Supervised } \\
\text { RT: Elastic band, } 50-70 \% \text { of 1RM, } \\
\text { upper extremities (lateral, anterior, } \\
\text { posterior) lower extremities (lift and } \\
\text { spread), weeks } 9-12\end{array}$ & $\begin{array}{l}\text { Pelvic floor } \\
\text { exercises }\end{array}$ & $\begin{array}{l}\text { General physical and } \\
\text { mental health } \mathrm{g}\end{array}$ \\
\hline $\begin{array}{l}\text { Segal et al., } \\
\text { (2003) [45] }\end{array}$ & $\begin{array}{c}155 \\
(82 / 73)\end{array}$ & $\begin{array}{l}\text { Tumour stage: T } 2-4 \\
\text { Metastatic disease: No } \\
\text { information } \\
\text { PCa treatment: ADT }\end{array}$ & 12 weeks & 3 times per week & $\begin{array}{c}\text { Supervised } \\
\text { RT: } 2 \text { sets, } 8-12 \text { reps, } 9 \text { exercises, } \\
60-70 \% \text { of } 1 \text { RM, } 5 \text { lbs increase when } \\
\text { able to complete }>12 \text { reps }\end{array}$ & $\begin{array}{l}\text { Waiting-list } \\
\text { protocol }\end{array}$ & $\begin{array}{l}\text { QoL }^{\mathrm{a}} \\
\text { Fatigue }^{\mathrm{d}}\end{array}$ \\
\hline $\begin{array}{l}\text { Segal et al., } \\
\text { (2009) [46] }\end{array}$ & $\begin{array}{c}121 \\
(40 / 40 / 41)\end{array}$ & $\begin{array}{l}\text { Tumour stage: T 1-4 } \\
\text { Metastatic disease: } \\
\text { None (excluded) } \\
\text { PCa treatment: RT and } \\
\text { ADT }\end{array}$ & 24 weeks & 3 times per week & $\begin{array}{c}\text { Supervised } \\
\text { AE: Weeks 1-4: } 50-60 \% \text { of VO2peak, } \\
\text { weeks 5-24: } 70-75 \% \text {, duration } 15 \\
\text { min progression } 5 \text { min every } 3 \\
\text { weeks until } 45 \text { min } \\
\text { RT: } 2 \text { sets, } 8-12 \text { reps of } 10 \text { exercises at } \\
60-70 \% \text { of } 1 \text { RM }\end{array}$ & $\begin{array}{l}\text { Waiting-list } \\
\text { protocol }\end{array}$ & $\begin{array}{c}\text { QoL }^{\mathrm{a}} \\
\text { Fatigue }^{\mathrm{d}} \\
\text { Cardiovascular fitness }^{\mathrm{n}} \\
\text { Lower body strength }^{\mathrm{w}} \\
{\text { Body fat mass }(\%)^{\mathrm{b}}}^{\mathrm{b}}\end{array}$ \\
\hline $\begin{array}{l}\text { Taaffe et al., } \\
\text { (2017) [47] }\end{array}$ & $\begin{array}{c}163 \\
(58 / 54 / 51)\end{array}$ & $\begin{array}{c}\text { Tumour stage: Gleason } \\
\text { mean } 7.8 \\
\text { Metastatic disease: } 7 \% \\
\text { of sample } \\
\text { PCa treatment: ADT }\end{array}$ & 24 weeks & $\begin{array}{c}\text { RT + IMP: } \\
2 \text { times per week } \\
\text { supervised, } 2 \text { times per } \\
\text { week home (just IMP) } \\
\text { RT + AE: } \\
2 \text { times per week }\end{array}$ & $\begin{array}{c}\text { RT + IMP supervised } \\
\text { RT: 2-4 sets (NI on reps), 6-12 RM, } 6 \\
\text { exercises } \\
\text { IMP: Ground reaction force 3,4-5,2 } \\
\text { times body weight } \\
\text { Home } \\
\text { IMP: As supervised } \\
\text { RT + AE supervised } \\
\text { RT: 2-4 sets (NI on reps), 6-12 RM, } 6 \\
\text { exercises } \\
\text { AE: } 20-30 \text { min, } 60-70 \% \text { of estimated } \\
\text { heart rate max } \\
\text { Home } \\
\text { AE: } 150 \text { min of moderate } \\
\text { cardiovascular fitness per week }\end{array}$ & $\begin{array}{l}\text { Waiting-list } \\
\text { protocol }\end{array}$ & $\begin{array}{l}\text { Fatigue }^{\mathrm{t}} \\
\text { Cardiovascular fitness }^{\mathrm{i}} \\
\text { Lower body strength }^{1} \\
{\text { Lean body mass }(\mathrm{kg})^{\mathrm{b}}}^{{ }^{\mathrm{b}}} \\
{\text { Body fat mass }(\mathrm{kg})^{\mathrm{b}}}\end{array}$ \\
\hline
\end{tabular}


Table 1. Cont.

\begin{tabular}{|c|c|c|c|c|c|c|c|}
\hline $\begin{array}{l}\text { Author } \\
\text { (Year) }\end{array}$ & $\begin{array}{l}\text { Sample } \\
\text { (Group } \\
\text { Randomised) }\end{array}$ & Population & $\begin{array}{l}\text { Intervention } \\
\text { Duration } \\
\text { (Follow-Up) }\end{array}$ & $\begin{array}{l}\text { Intervention } \\
\text { Frequency }\end{array}$ & Intervention Description & Control Group & Outcomes of Interest \\
\hline $\begin{array}{l}\text { Taaffe et al., } \\
\text { (2019) [48] }\end{array}$ & $\begin{array}{c}104 \\
(54 / 50)\end{array}$ & $\begin{array}{c}\text { Tumour stage: Gleason } \\
\text { mean } 7.6 \\
\text { Metastatic disease: } \\
\text { None, exclusion criteria } \\
\text { PCa treatment: ADT }\end{array}$ & 24 weeks & $\begin{array}{l}3 \text { times per week } \\
\text { supervised, } 2 \text { times per } \\
\text { week home }\end{array}$ & $\begin{array}{c}\text { Supervised } \\
\text { AE: Treadmill, rower or bike 60-80\% } \\
\text { heart rate max } \\
\text { RT: Major muscle groups 2-4 sets, } \\
\text { 6-12 reps } \\
\text { IMP: Hopping, skipping, leaping } \\
\text { and drop jump (GRF 3.4-5.2 times } \\
\text { body weight) } \\
\text { Home } \\
\text { Walking and modified IMP }\end{array}$ & $\begin{array}{l}\text { Waiting-list } \\
\text { protocol }\end{array}$ & $\begin{array}{l}\text { Lean body mass }(\mathrm{kg})^{\mathrm{b}} \\
\text { Body fat mass }(\mathrm{kg})^{\mathrm{b}}\end{array}$ \\
\hline $\begin{array}{l}\text { Uth et al. } \\
\text { (2014) [49] }\end{array}$ & $\begin{array}{c}57 \\
(29 / 28)\end{array}$ & $\begin{array}{c}\text { Tumour stage: Gleason } \\
\text { mean } 7.9 \\
\text { Metastatic disease: } \\
\text { Bone metastases } 19 \% \text { of } \\
\text { sample } \\
\text { PCa treatment: ADT }\end{array}$ & 12 weeks & $\begin{array}{l}2 \text { times per week } \\
\text { (weeks 1-8), } 3 \text { times per } \\
\text { week (weeks 9-12) }\end{array}$ & $\begin{array}{c}\text { Supervised } \\
\text { AE: } 2 \times 15 \text { min of } 5-7 \text { a-side football } \\
\text { (weeks } 1-4), 3 \times 15 \text { min of } 5-7 \text { a-side } \\
\text { football (weeks } 5-12 \text { ) }\end{array}$ & $\begin{array}{l}\text { Waiting-list } \\
\text { protocol }\end{array}$ & 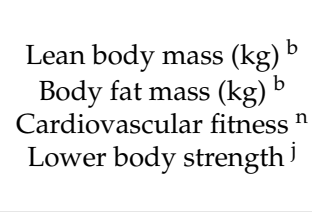 \\
\hline $\begin{array}{l}\text { Windsor et al., } \\
\text { (2004) [50] }\end{array}$ & $\begin{array}{c}66 \\
(33 / 33)\end{array}$ & $\begin{array}{l}\text { Tumour stage: T 1-2 (51 } \\
\text { out of } 66) \\
\text { Metastatic disease: } \\
\text { Unclear } \\
\text { PCa treatment: RT }\end{array}$ & 4 (8) weeks & 3 times per week & $\begin{array}{l}\text { Home } \\
\text { AE: } 30 \text { min of walking at moderate } \\
\text { intensity }(60-70 \% \text { of heart rate max) }\end{array}$ & Usual care & Fatigue $^{\mathrm{m}}$ \\
\hline $\begin{array}{l}\text { Wall et al., } \\
\text { (2017) [51] }\end{array}$ & $\begin{array}{c}97 \\
(50 / 47)\end{array}$ & $\begin{array}{c}\text { Tumour stage: Gleason } \\
\text { mean } 8.0 \\
\text { Metastatic disease: } \\
\text { None (bone metastases } \\
\text { excluded) } \\
\text { PCa treatment: ADT }\end{array}$ & 24 weeks & 2 times per week & $\begin{array}{c}\text { Supervised } \\
\text { RT: } 6 \text { exercises, weeks } 1-4: 2 \text { sets, } 12 \\
\text { reps, weeks 5-8: } 3 \text { sets, } 10 \text { reps, } \\
\text { weeks 9-12: } 3 \text { sets, } 8 \text { reps, weeks } \\
\text { 13-16: ? } \\
\text { AE: } 20-30 \text { min, } 70-90 \% \text { of VO2peak } \\
\text { Home } \\
150 \text { min per week } 70-90 \% \text { of } \\
\text { VO2peak }\end{array}$ & Usual care & 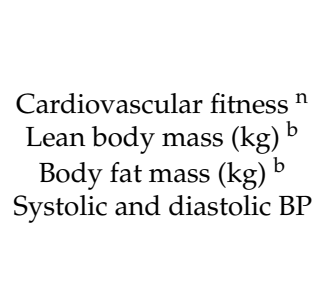 \\
\hline
\end{tabular}


Table 1. Cont.

\begin{tabular}{|c|c|c|c|c|c|c|c|}
\hline $\begin{array}{l}\text { Author } \\
\text { (Year) }\end{array}$ & $\begin{array}{l}\text { Sample } \\
\text { (Group } \\
\text { Randomised) }\end{array}$ & Population & $\begin{array}{l}\text { Intervention } \\
\text { Duration } \\
\text { (Follow-Up) }\end{array}$ & $\begin{array}{l}\text { Intervention } \\
\text { Frequency }\end{array}$ & Intervention Description & Control Group & Outcomes of Interest \\
\hline $\begin{array}{l}\text { Winters- } \\
\text { Stone et al., } \\
\text { (2014) [52] }\end{array}$ & $\begin{array}{c}51 \\
(29 / 22)\end{array}$ & $\begin{array}{l}\text { Tumour stage: Unclear } \\
\text { Metastatic disease: } \\
27.6 \% \text { of intervention } \\
\text { sample, } 13.6 \% \text { of control } \\
\text { sample } \\
\text { PCa treatment: ADT }\end{array}$ & 52 weeks & $\begin{array}{c}2 \text { times per week } \\
\text { supervised }+1 \text { time per } \\
\text { week home }\end{array}$ & $\begin{array}{c}\text { Supervised } \\
\text { RT: Lower body: } 2-3 \text { sets } \\
\text { progression from } 12-14 \text { RM, } \\
\text { 12-14 reps to 6-8 RM, 6-8 reps } \\
\text { Upper body: } 2-3 \text { sets, } 8-12 \text { reps, } \\
\text { progressing from } 0-2 \% \text { to } 9-10 \% \text { of } \\
\text { body weight } \\
\text { IMP: Jumps: } 10 \text { reps, progressing } \\
\text { from } 3 \text { to } 10 \text { sets } \\
\text { Home } \\
\text { As supervised }\end{array}$ & Stretching protocol & $\begin{array}{c}\text { Lean body mass }(\mathrm{kg})^{\mathrm{b}} \\
{\text { Body fat mass }(\mathrm{kg})^{\mathrm{b}}}^{\text {Fatigue }^{\mathrm{x}}}\end{array}$ \\
\hline $\begin{array}{l}\text { Winters- } \\
\text { Stone et al., } \\
\text { (2016) [53] }\end{array}$ & $\begin{array}{c}64 \\
(32 / 32)\end{array}$ & $\begin{array}{c}\text { Tumour stage: Unclear } \\
\text { Metastatic disease: } 9 \% \\
\text { of sample } \\
\text { PCa treatment: } \\
\text { Currently on ADT: } \\
\text { Intervention }(22 \%) \text {, } \\
\text { control }(13 \%)\end{array}$ & 24 weeks & 2 times per week & $\begin{array}{c}\text { Supervised } \\
\text { RT exercising with spouse: } \\
\text { 8-10 exercises, } 8-15 \text { reps, } \\
\text { progressing from } 15 \text { to } 8 \text { RM }\end{array}$ & $\begin{array}{l}\text { Waiting-list } \\
\text { protocol }\end{array}$ & $\begin{array}{l}\text { Lean body mass }(\mathrm{kg})^{\mathrm{b}} \\
\text { Body fat mass }(\mathrm{kg})^{\mathrm{b}} \\
\text { General physical and } \\
\text { mental health } \mathrm{g} \\
\text { Lower body strength }\end{array}$ \\
\hline
\end{tabular}

The column "Intervention description" contains both supervised and home-delivered exercise characteristics. PCa, prostate cancer, AE, aerobic exercise, RT, resistance training, BP, blood pressure, QoL, quality of life, ADT, androgen-deprivation therapy, BW, body weight, IMP, impact training, NI, no information, RM, repetition maximum, reps, repetition; ${ }^{a}$ FACT-P, Functional Assessment of Cancer Therapy-Prostate, ${ }^{\mathrm{b}}$ DXA, dual-energy X-ray absorption, ${ }^{\mathrm{c}}$ SF-12, 12-Item Short Form Health Survey, ${ }^{\mathrm{d}}$ FACT-F, Functional Assessment of Cancer Therapy-Fatigue, e Bruce ramp protocol, ${ }^{\mathrm{f}}$ EQ5D questionnaire, ${ }^{\mathrm{g}}$ SF-36, 36-Item Short Form Health Survey, ${ }^{\mathrm{h}}$ MFSI-SF, Multidimensional Fatigue Symptom Inventory-Short Form, ${ }^{\mathrm{i}}$ 400 $\mathrm{m}$ walk test, ${ }^{\mathrm{j}}$ 1RM leg extension, ${ }^{\mathrm{k}}$ FACIT-F, Functional Assessment of Chronic Illness Therapy-Fatigue, ${ }^{1} 1 \mathrm{RM}$ leg press, ${ }^{\mathrm{m}}$ BFI, Brief Fatigue Inventory, ${ }^{\mathrm{n}}$ VO2max/peak (incremental cycling test with pulmonary gas exchange measurement), ${ }^{\circ}$ EORCT QLQ-C30 (global), European Organization for Research and Treatment of Cancer Quality of Life Questionnaire-C30, p 6 min walk-test, ${ }^{r}$ Piper Fatigue Scale, ${ }^{s}$ Timed 5 reps of chair sit-to-stand, ${ }^{t}$ EORCT QLQ-C30 (Fatigue), European Organization for Research and Treatment of Cancer Quality of Life Questionnaire-C30, u shuttle walk test, ${ }^{\mathrm{V}}$ FSS, Fatigue Severity Scale, ${ }^{\mathrm{w}} 8 \mathrm{RM}$ leg press, ${ }^{\mathrm{N}}$ Schwartz Cancer Fatigue Scale, and BIA, bioelectrical impedance analysis. 


\subsection{Risk of Bias within Studies}

Supplementary Material File S2 contains the risk of bias summary table, while the risk of bias with reason is available in Supplementary Material File S3.

\subsection{Synthesis of Results}

Seventeen trials involving 1361 participants measured cancer-specific QoL [22-24,27,31,35,36,38-43,45,46,54]. Segal et al. [46] presented two intervention arms for comparison, providing a total of 16 comparisons eligible for meta-analysis. The metaanalysis showed a borderline significant pooled small effect estimate (SMD $=0.10,95 \% \mathrm{CI}$ $-0.01-0.22$ ) (Figure 2) in favour of exercise interventions and with no statistical heterogeneity (I-squared $=0.0 \%, p=0.718$ ). No significant difference was seen between exercise modalities, and no modality alone yielded significant effect estimates (Supplementary Material File S4, Figure S1). Likewise, we found no difference between the assessment instruments (Supplementary Material File S4, Figure S2). There was no indication of publication bias detected in the funnel plot or in the Egger's test (Supplementary Material File S4, Figure S3).

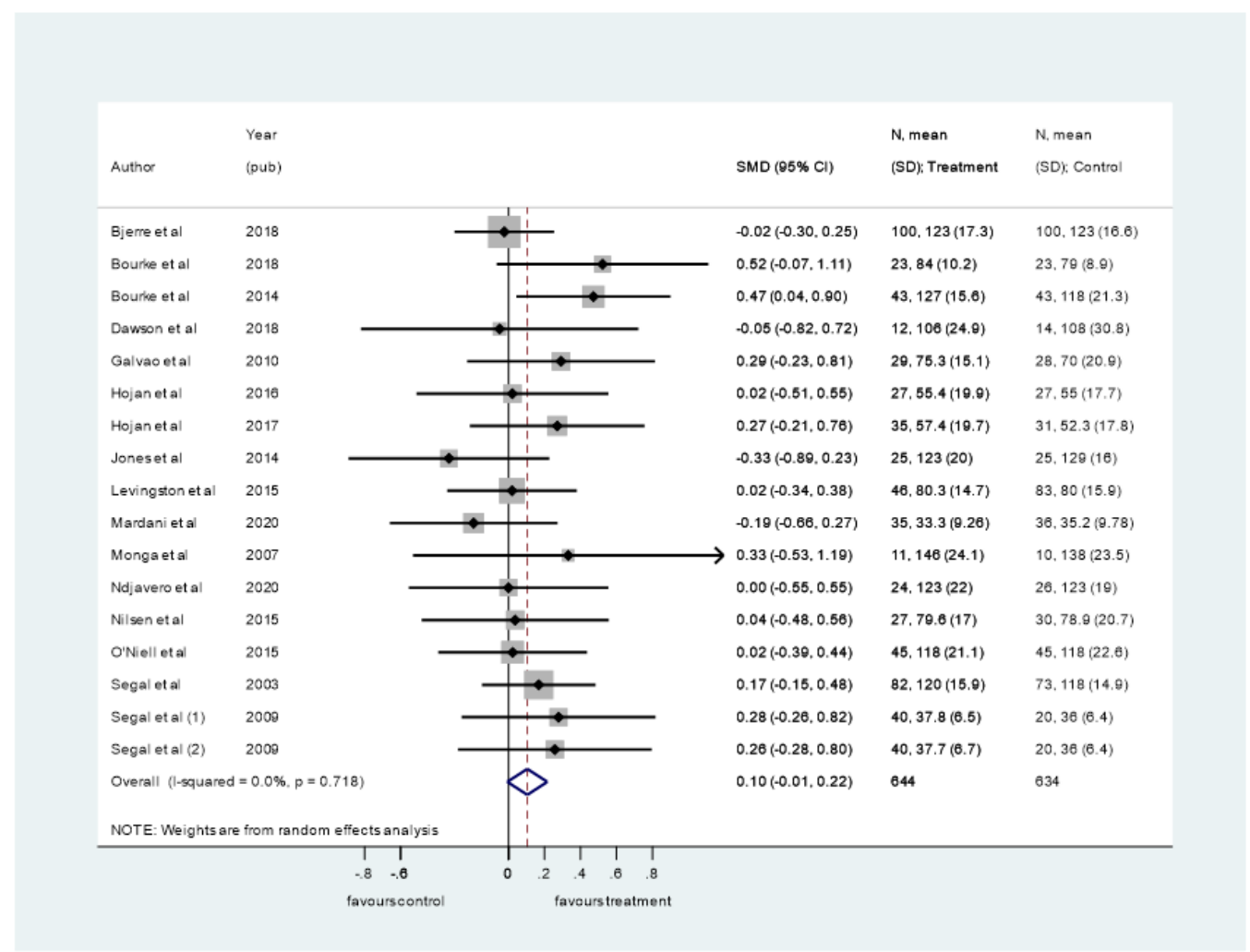

Figure 2. Pooled standard mean difference (SMD) on cancer-specific quality of life comparing exercise interventions with usual care or control in men with prostate cancer. A random effects model of DerSimonian and Laird, with estimate of heterogeneity from the Mantel-Haenszel model was used.

Twenty-four trials involving 1618 participants measured cardiovascular fitness [23-27,29-33,35-43,46,47,49-51]. Segal et al. [46] and Taaffe et al. [47] each presented two intervention arms for comparison. Meta-analysis was feasible for 22 trials, yielding 24 comparisons. Windsor et al. [50] only reported results on cardiovascular fitness after four weeks of intervention (not eight weeks, which was the end of the intervention). Focht et al. [30] reported only change scores, and post-intervention measurement could not be calculated as baseline measurements were not reported. Consequently, data from these studies were excluded from the meta-analysis.

Meta-analysis demonstrated an overall significant, moderate to large effect estimate in favour of exercise (SMD $=0.46,95 \%$ CI 0.34-0.56) (Figure 3) and moderate heterogeneity 
(I-squared $=26,8 \%, p=0.108$ ). When comparing exercise modalities, all yielded a significant effect with aerobic exercise as the superior modality, corresponding to a large effect size (SMD $=0.60,95 \%$ CI 0.29-0.90) (Supplementary Material File S4, Figure S5). There was no difference between using direct measures of cardiovascular fitness (VO2 peak) or a submaximal measure (e.g., $400 \mathrm{~m}$ walk) (Supplementary Material File S4, Figure S6). There was no indication for publication bias detected in the funnel plot or the Egger's test (Supplementary Material File S4, Figure S7).

\begin{tabular}{|c|c|c|c|c|c|}
\hline Author & $\begin{array}{l}\text { Year } \\
\text { (pub) }\end{array}$ & & SMD $(95 \% \mathrm{Cl})$ & $\begin{array}{l}\text { N. mean } \\
\text { (SD): Treatment }\end{array}$ & $\begin{array}{l}\text { N. mean } \\
\text { (SD): Control }\end{array}$ \\
\hline Bourke otal & 2014 & & $0.78(0.32 .1 .20)$ & $43,470(127)$ & $42,375(137)$ \\
\hline Bourke et al & 2018 & & $0.46(-0.12,1.05)$ & $23.841(158)$ & $23.574(130)$ \\
\hline Cormie etal & 2013 & & $0.93(0.00 .1 .88)$ & $10,-247(32.8)$ & $10 .-287(50.5)$ \\
\hline Cormie etal & 2015 & & $0.00(-0.49,0.49)$ & $32.22 .7(3.8)$ & $31.22 .7(3.6)$ \\
\hline Dawsonetal & 2018 & & $0.44(-0.34 .1 .23)$ & $11,-228(46.7)$ & $15,-258(79)$ \\
\hline Eriksen etal & 2017 & & $0.78(-0.16 .1 .72)$ & $14.32(7)$ & 7.27 (5) \\
\hline Galvao et al & 2010 & & $0.34(-0.18,0.80)$ & $29,-257(37)$ & $28,-270(33.8)$ \\
\hline Galvao et al & 2014 & & $0.07(-0.32 .0 .48)$ & $50,-270(51,6)$ & $50,-274(51.6)$ \\
\hline Galvao et al & 2018 & & $0.11(-0.46 .0 .68)$ & $22 .-245(32.9)$ & $26,-249(41)$ \\
\hline Hojan etal & 2016 & & $0.29(-0.25,0.82)$ & $27.441(68.4)$ & $27.422(67.5)$ \\
\hline Hojan etal & 2017 & 8 & $1.02(0.51 .1 .54)$ & $35.457(77.4)$ & $31,378(76.3)$ \\
\hline Hvid et al & 2016 & & $0.85(-0.30 .1 .61)$ & $12.32 .0(9.2)$ & $7.27 .4(4.9)$ \\
\hline Jones et al & 2014 & & $0.08(-0.49 .0 .82)$ & $25.24 .2(5.3)$ & $25.23 .9(4.4)$ \\
\hline Levingston et al & 2015 & & $0.58(0.20 .0 .93)$ & $53,609(81.8)$ & $86.567(67.9)$ \\
\hline Monga et al & 2007 & & $1.52(0.54 .2 .50)$ & $11,9.8(.8)$ & $10.7 .2(2.3)$ \\
\hline Ndjavero etal & 2020 & & $0.54(-0.03 .1 .10)$ & $24.23 .2(5.1)$ & $26.20 .4(5.3)$ \\
\hline Nilsenetal & 2015 & & $0.32(-0.22,0.80)$ & $25,804(204)$ & $29.741(189)$ \\
\hline O'Niell etal & 2015 & $\rightarrow$ & $1.03(0.59 .1 .47)$ & $45.553(78.8)$ & $45,474(75.8)$ \\
\hline Segal et al (1) & 2009 & & $0.33(-0.21,0.87)$ & $40.29 .8(6.4)$ & $20,27.8(5.1)$ \\
\hline Segal et al (2) & 2009 & & $0.24(-0.29 .0 .77)$ & $40.28 .1(8.8)$ & $21,27.8(5.1)$ \\
\hline Taaffe et al & 2019 & & $0.46(0.08 .0 .85)$ & $54,-242(37.8)$ & $49,-264(56.9)$ \\
\hline Taaffe et al (1) & 2017 & & $0.24(-0.24 .0 .72)$ & $57,-264(47.7)$ & $24 .-276(53.8)$ \\
\hline Taaffe et al (2) & 2017 & & $0.47(-0.02,0.06)$ & $54,-255(41.7)$ & $24,-278(53.8)$ \\
\hline Uth et al & 2012 & & $0.42(-0.15,0.98)$ & $26.28 .7(5.2)$ & $23,26.9(3)$ \\
\hline Wall et al & 2017 & & $0.81(0.20 .1 .01)$ & $50.25 .4(5.7)$ & $47.22 .2(4.8)$ \\
\hline Overall (I-squar & $e d=26.8 \%, p=0.108)$ & $\diamond$ & $0.48(0.34 .0 .59)$ & 812 & 700 \\
\hline \multicolumn{6}{|c|}{ NOTE: Weights are from random effects analysis } \\
\hline & 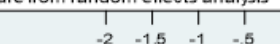 & $\begin{array}{ll}1 & 1 \\
15 & 2\end{array}$ & & & \\
\hline
\end{tabular}

Figure 3. Pooled standard mean difference (SMD) on cardiovascular fitness comparing exercise interventions with usual care or control in men with prostate cancer. A random effects model of DerSimonian and Laird, with estimate of heterogeneity from the Mantel-Haenszel model, was used.

Supplementary Material File S5 provides the forest plots for the remaining outcomes. Meta-analysis demonstrated a small significant overall effect of exercise on fatigue (SMD $=0.27$ 95\% CI 0.13-0.40) (Supplementary Material File S5, Figure S10). Two Hojan et al. $[35,36]$ trials were excluded from the meta-analysis in relation to fatigue as they reported extreme effect estimates and the corresponding author did not respond when contacted about this. For the outcome lower body strength, a small to moderate overall effect was seen (SMD $=0.44,95 \%$ CI 0.26-0.62) (Supplementary Material File S5, Figure S12), while resistance exercise had a moderate to large effect (SMD $=0.6695 \%$ CI $0.27-1.04$ ) (Supplementary Material File S5, Figure S13). Exercise showed no significant overall effect on lean body mass (WMD $=0.23 \mathrm{~kg}, 95 \%$ CI $-0.51-0.97$ ) (Supplementary Material File S5, Figure S14); however, resistance exercise did yield a significant effect (WMD $=2.03 \mathrm{~kg}, 95 \%$ CI 0.30-3.75) (Supplementary Material File S5, Figure S15). For the outcome whole-body fat mass, exercise demonstrated an overall significant reduction (WMD $=-1.13 \mathrm{~kg}, 95 \% \mathrm{CI}$ -1.92--0.33) (Supplementary Material File S5, Figure S16), with no difference between modalities (Supplementary Material File S5, Figure S17). No effect was seen for exercise overall concerning general physical health (WMD $=1.40,95 \%$ CI $-0.58-3.38$ ) (Supplementary Material File S5, Figure S19); however, resistance exercise alone showed a significant effect (WMD $=2.61,95 \%$ CI 0.44-4.79) (Supplementary Material File S5, Figure S20). For general mental health, an overall effect was seen (WMD $=2.58,95 \%$ CI 1.33-3.84) (Supplemen- 
tary Material File S5, Figure S22), with combined aerobic and resistance training as the superior modality (WMD $=3.64,95 \%$ CI 1.33-5.94) (Supplementary Material File S5, Figure S23). Lastly, a small reduction in both systolic and diastolic blood pressure was observed equal to $-2.84 \mathrm{mmHg}$ (95\% CI: - 6.34-0.67) (Supplementary Material File S5, Figures S24 and S25) and $-1.80 \mathrm{mmHg}(95 \% \mathrm{CI}-3.82-0.23)$ (Supplementary Material File S5, Figures S26 and S27), respectively.

\section{Discussion}

\subsection{Summary of Evidence}

In this systematic review of RCTs reporting on the effect of exercise for men with prostate cancer, a borderline small positive effect was found on cancer-specific QoL, and a moderate to large positive effect was found on cardiovascular fitness. Furthermore, beneficial effects of exercise were found in the outcomes: lower body strength, wholebody fat mass, general mental health, systolic and diastolic blood pressure, and fatigue. However, no significant beneficial effects were identified on lean body mass and general physical health.

The beneficial effects of exercise on cancer-specific QoL are in line with a previous systematic review by Bourke et al. [7]; however, the magnitude of previous reported results was larger compared with this review. One possible explanation is that Bourke et al. based their conclusion on a sensitivity analysis that only included three studies assessed as high-quality studies. Our results do not provide evidence that one specific exercise modality is superior in improving cancer-specific QoL. This is supported by existing headto-head evidence in two trials. Specifically, Santa Mina et al. [78], who compared aerobic exercise and resistance exercise, found no significant difference in health-related QoL in men with prostate cancer. Additionally, Segal et al. [46] found no significant effect of group assignment (aerobic exercise versus resistance exercise) on either prostate-specific QoL or cancer-specific QoL. Most of the trials in this systematic review reported no effects or small effects on cancer-specific QoL. Only one trial, by Bourke et al. [23], found significant and clinically relevant changes in favour of exercise when combining exercise with nutrition and behavioural support, suggesting that exercise alone may not be sufficient to improve cancerspecific QoL. Accordingly, researchers intending to improve QoL among men diagnosed with prostate cancer might investigate the potential mediating effect of combining exercise with other therapeutic interventions.

We found a small significant effect of exercise on fatigue, which is in line with findings from a recent review by Lopez et al. [10]. In contrast, a large systematic review with results that lacked statistical significance suggested that caution is warranted in terms of making any definitive, all-encompassing conclusions on the benefits of exercise on cancer-related fatigue [79]. Our results do not provide evidence that one specific exercise modality is superior in improving fatigue, which is supported by Taaffe et al. [47] who examined different exercise modalities on fatigue in men diagnosed with prostate cancer. Fatigue is believed to be the most frequent and influential debilitating symptom of prostate cancer and its treatment [80], and is a prevalent indicator of the QoL of cancer patients [81]. Thus, the lack of effect on fatigue may partially explain the modest effects documented in cancerspecific QoL in the current review. In addition, the modest changes in cancer-specific QoL and fatigue may also be due to floor and ceiling effects, as most studies did not aim to recruit participants with clinical levels of fatigue and/or suboptimal QoL. In this regard, a comprehensive individual patient data meta-analysis including 4519 cancer patients investigated the moderator effects of baseline values on various exercise outcomes [82] and found that the effect of exercise interventions post-cancer treatment on health-related QoL and fatigue appeared to be greater in patients with worse baseline values. Because no or marginal room for improvement exists in the case of high QoL and/or low fatigue at baseline, the beneficial effects of exercise may therefore be underestimated. Further research to investigate the effect of exercise in a target prostate cancer population reporting high fatigue and low QoL at baseline appears warranted. 
The current meta-analysis found exercise to be useful in improving cardiovascular fitness and in lowering whole-body fat mass and blood pressure, which is in line with recent evidence on metabolic health in a prostate cancer population on ADT [11]. All exercise modalities were found to be effective in improving cardiovascular fitness with aerobic exercise as yielding large effect estimates. This is important in the light of evidence of a significantly increased risk of men with prostate cancer developing metabolic diseases, mostly due to ADT treatment [2]. In a recent study, cardiovascular fitness was inversely associated with the overall metabolic syndrome score [83]; hence, together with the improvements seen in the current study on isolated metabolic risk factors (i.e., blood pressure and body distribution), the benefits of exercise on cardiovascular fitness could be seen as highly clinically relevant for improving metabolic health in men with prostate cancer.

It is worth noting that the included studies assessed cardiovascular fitness by means of either a direct measure of maximum/peak oxygen uptake (most frequently the incremental cycling test while analysing gas exchange) or a sub-maximal performance test (most frequently the $400 \mathrm{~m}$ walk test or 6 min walk test). Most studies investigating aerobic exercise used a direct measure of maximum oxygen uptake $[29,37,38,46,49]$, while studies investigating resistance exercise or a combination of resistance and aerobic exercise used a submaximal performance test. Such an increase in muscle strength in the lower body may have a positive effect on walking endurance without increasing oxygen uptake. However, submaximal walking tests (such as 6 min walk) have been found valid and reliable for evaluating cardiorespiratory fitness in patients with cancer [84]. Lastly, it must be acknowledged that improvements in metabolic health cannot be sustained without continuous adherence to exercise or physical activity, which is why it is imperative to conduct more studies examining and promoting the longer-term effects of exercise adoption in men diagnosed with prostate cancer.

The aim of the current systematic review was to examine possible differential effects across specific exercise modalities. We chose to stratify exercise interventions into aerobic exercise, resistance exercise, or a combination of the two. Our findings indicate that the choice of modality may be important when targeting physiological outcomes. Thus, not surprisingly, whereas aerobic exercise was superior in improving cardiovascular fitness (Supplementary Material File S4, Figure S5), resistance exercise appeared superior in improving lower body strength (Supplementary Material File S5, Figure S13) and lean body mass (Supplementary Material File S5, Figure S15). However, exercise modalities did not show a mediating effect on PROMs such as cancer-specific QoL (Supplementary Material File S4, Figure S1) and fatigue (Supplementary Material File S5, Figure S11). This observation is supported by a position statement from the Exercise and Sports Science in Australia association, which concludes that the appropriate exercise prescription should be targeted and individualised according to patient- and cancer-specific considerations [85]. However, this is often a challenge in exercise RCTs because they follow a strict intervention protocol to ensure internal validity and to reduce the potential risk of bias. We encourage future research to allow a more flexible intervention protocol to investigate the effectiveness of individually tailored exercise according to physical needs and patient preferences.

\subsection{Limitations}

Some caution is warranted when interpreting the results of the current review. First, there was considerable within-study heterogeneity in the trial populations regarding cancer stage and treatment, which meant that conducting relevant subgroup analyses were not possible. Furthermore, the generalisability may be limited to patients treated with ADT, and likely those most motivated in terms of adherence to exercise, which may not be representative of the background population of men with prostate cancer. Caution is needed when transferring the results to men diagnosed with non-aggressive prostate cancer who are under active surveillance or with advanced prostate cancer with metastatic disease, as these groups are underrepresented in the current review. We encourage researchers to share raw data on patient characteristics (tumour histology, prior treatment, treatment response, and 
metastatic spread) to allow relevant subgroup analysis on individual patient's data in future meta-analytic procedures. Second, even though exercise interventions were categorised into aerobic, resistance or combined exercise, the content varied across studies in relation to frequency, duration and intensity. We did not aim to investigate the moderating effects of these factors. However, most included trials used an exercise protocol lasting between 12 and 24 months with a frequency of two to three sessions per week [86]. This correlates with existing guidelines, which recommend a minimum of 12 weeks of combined aerobic and resistance exercise twice a week to meet the health challenges faced by prostate cancer treated with ADT. Future research may focus on the optimal frequency and intensity of exercise and how to best promote and support the long-term adoption of exercise behaviour in this population.

Furthermore, some studies combined exercise with other behavioural interventions such as dietary and cognitive behavioural therapy. The current review did not include trials investigating low-intensity exercise (e.g., qigong, tai chi [87] and yoga [88]), which may be a beneficial modality in improving QoL and fatigue. Furthermore, several trials reported no or insufficient information on adherence and fidelity, making it uncertain to what degree patients followed prescribed exercise. A recent systematic review stated that most studies investigating exercise interventions for prostate cancer survivors report adherence information on the frequency of exercise sessions completed but fail to report fidelity information on exercise intensity and duration [89].

The strengths of this systematic review and meta-analysis include a comprehensive search strategy, systematic risk of bias evaluation and a prespecified, systematic data synthesis.

\section{Conclusions}

In summary, the results of this comprehensive systematic review and meta-analysis suggest that men with prostate cancer can benefit from exercise. We found positive effects on metabolic health, specifically increased cardiovascular fitness, and reduced whole-body fat mass and blood pressure. Furthermore, exercise appeared to have a small positive effect on cancer-specific QoL and fatigue. These findings indicate that using exercise complementarily to prostate cancer treatment may mitigate the development of metabolic disease. Future research should focus on combining exercise with other behavioural interventions and/or should target patients with suboptimal baseline values who are deemed to be most likely to benefit from exercise. The choice of exercise modality may be important when targeting physiological outcomes; however, no modality appears to be superior when addressing PROMs.

Supplementary Materials: The following supporting information can be downloaded at: https: / / www.mdpi.com/article/10.3390/ijerph19020972/s1, File S1: Search Strategy PubMed, File S2: Risk of Bias summary table, File S3: Risk of Bias with reason, File S4: Forest plots of additional analyses to QoL and cardiovascular fitness, File S5: Forest plots of remaining outcomes, File S6: PRISMA 2020 Checklist. Figure S1: Pooled standard mean difference (SMD) on Cancer-specific quality of life comparing exercise interventions with usual care or control in men with prostate cancer stratified after exercise modality. A random effects model of DerSimonian \& Laird, with estimate of heterogeneity from the Mantel-Haenszel model was used. AE: aerobic exercise, RE: resistance exercise. Figure S2: Pooled standard mean difference (SMD) on Cancer-specific quality of life comparing exercise interventions with usual care or control in men with prostate cancer stratified after assessment instrument. A random effects model of DerSimonian \& Laird, with estimate of heterogeneity from the Mantel-Haenszel model was used. Figure S3: Funnel plot with pseudo 95\% confidence limit onCancer-specific quality of life in men with prostate cancer. Egger's test showed no small-study effects $(\mathrm{P}=0.729)$, Figure S4: Pooled standard mean difference (SMD) for change score values on Cancer-specific quality of life comparing exercise interventions with usual care or control in men with prostate cancer. A random effects model of DerSimonian \& Laird, with estimate of heterogeneity from the Mantel-Haenszel model was used, Figure S5: Pooled standard mean difference (SMD) on Cardiovascular fitness comparing exercise interventions with usual care or control in men with prostate cancer stratified after exercise modality. A random effects model of DerSimonian \& 
Laird, with estimate of heterogeneity from the Mantel-Haenszel model was used, Figure S6: Pooled standard mean difference (SMD) on Cardiovascular fitness comparing exercise interventions with usual care or control in men with prostate cancer stratified after assessment instrument. A random effects model of DerSimonian \& Laird, with estimate of heterogeneity from the Mantel-Haenszel model was used, Figure S7: Funnel plot with pseudo 95\% confidence limit on Cardiovascular function in men with prostate cancer. Egger's test showed no small-study effects $(\mathrm{P}=0.647)$, Figure S8: Pooled standard mean difference (SMD) for change score values on Cardiovascular fitness comparing exercise interventions with usual care or control in men with prostate cancer. A random effects model of DerSimonian \& Laird, with estimate of heterogeneity from the Mantel-Haenszel model was used, Figure S9: Pooled standard mean difference (SMD) on Fatigue comparing exercise interventions with usual care or control in men with prostate cancer. A random effects model of DerSimonian \& Laird, with estimate of heterogeneity from the Mantel-Haenszel model was used, Figure S10: Pooled standard mean difference (SMD) on Fatigue comparing exercise interventions with usual care or control in men with prostate cancer. A random effects model of DerSimonian \& Laird, with estimate of heterogeneity from the Mantel-Haenszel model was used. NOTE: Hojan et al. 2016 and 2017 was excluded from this meta-analysis, Figure S11: Pooled standard mean difference (SMD) on Fatigue comparing exercise interventions with usual care or control in men with prostate cancer stratified after exercise modality. A random effects model of DerSimonian \& Laird, with estimate of heterogeneity from the Mantel-Haenszel model was used. NOTE: Hojan et al. 2016 and 2017 was excluded from this meta-analysis, Figure S12: Pooled standard mean difference (SMD) on Lower body strength comparing exercise interventions with usual care or control in men with prostate cancer. A random effects model of DerSimonian \& Laird, with estimate of heterogeneity from the Mantel-Haenszel model was used, Figure S13: Pooled standard mean difference (SMD) on Lower body strength comparing exercise interventions with usual care or control in men with prostate cancer stratified after exercise modality. A random effects model of DerSimonian \& Laird, with estimate of heterogeneity from the Mantel-Haenszel model was used, Figure S14: Pooled standard mean difference (SMD) on Lean body mass comparing exercise interventions with usual care or control in men with prostate cancer. A random effects model of DerSimonian \& Laird, with estimate of heterogeneity from the Mantel-Haenszel model was used, Figure S15: Pooled standard mean difference (SMD) on Lean body mass comparing exercise interventions with usual care or control in men with prostate cancer stratified after exercise modality. A random effects model of DerSimonian \& Laird, with estimate of heterogeneity from the Mantel-Haenszel model was used, Figure S16: Pooled standard mean difference (SMD) on Whole body fat comparing exercise interventions with usual care or control in men with prostate cancer. A random effects model of DerSimonian \& Laird, with estimate of heterogeneity from the Mantel-Haenszel model was used, Figure S17: Pooled standard mean difference (SMD) on Whole body fat comparing exercise interventions with usual care or control in men with prostate cancer stratified after exercise modality. A random effects model of DerSimonian \& Laird, with estimate of heterogeneity from the Mantel-Haenszel model was used, Figure S18: Pooled standard mean difference (SMD) on General physical health comparing exercise interventions with usual care or control in men with prostate cancer. A random effects model of DerSimonian \& Laird, with estimate of heterogeneity from the Mantel-Haenszel model was used, Figure S19: Pooled standard mean difference (SMD) on General physical health comparing exercise interventions with usual care or control in men with prostate cancer. A random effects model of DerSimonian \& Laird, with estimate of heterogeneity from the Mantel-Haenszel model was used. NOTE: Park et al. 2012 was excluded from the meta-analysis, Figure S20: Pooled standard mean difference (SMD) on General physical health comparing exercise interventions with usual care or control in men with prostate cancer stratified after exercise modality. A random effects model of DerSimonian \& Laird, with estimate of heterogeneity from the Mantel-Haenszel model was used, Figure S21: Pooled standard mean difference (SMD) on General mental health comparing exercise interventions with usual care or control in men with prostate cancer. A random effects model of DerSimonian \& Laird, with estimate of heterogeneity from the Mantel-Haenszel model was used, Figure S22: Pooled standard mean difference (SMD) on General mental health comparing exercise interventions with usual care or control in men with prostate cancer. A random effects model of DerSimonian \& Laird, with estimate of heterogeneity from the Mantel-Haenszel model was used. NOTE: Park et al. 2012 was excluded from the meta-analysis, Figure S23: Pooled standard mean difference (SMD) on General mental health comparing exercise interventions with usual care or control in men with prostate cancer stratified after exercise modality. A random effects model of 
DerSimonian \& Laird, with estimate of heterogeneity from the Mantel-Haenszel model was used, Figure S24: Pooled standard mean difference (SMD) on Systolic blood pressure comparing exercise interventions with usual care or control in men with prostate cancer. A random effects model of DerSimonian \& Laird, with estimate of heterogeneity from the Mantel-Haenszel model was used, Figure S25: Pooled standard mean difference (SMD) on Systolic blood pressure comparing exercise interventions with usual care or control in men with prostate cancer stratified after exercise modality. A random effects model of DerSimonian \& Laird, with estimate of heterogeneity from the MantelHaenszel model was used, Figure S26: Pooled standard mean difference (SMD) on Diastolic blood pressure comparing exercise interventions with usual care or control in men with prostate cancer. A random effects model of DerSimonian \& Laird, with estimate of heterogeneity from the MantelHaenszel model was used, Figure S27: Pooled standard mean difference (SMD) on Diastolic blood pressure comparing exercise interventions with usual care or control in men with prostate cancer stratified after exercise modality. A random effects model of DerSimonian \& Laird, with estimate of heterogeneity from the Mantel-Haenszel model was used.

Author Contributions: M.F.A. and E.D.B. initiated and planned the study and conducted the data collection and analyses. M.F.A. wrote the first draft of the manuscript, which was critically reviewed and revised by E.D.B. and J.M. All authors have read and agreed to the published version of the manuscript.

Funding: This research received no external funding.

Institutional Review Board Statement: Not applicable.

Informed Consent Statement: Not applicable.

Data Availability Statement: Please contact the corresponding author to request data.

Acknowledgments: We thanks Anders Larsen, information specialist, for help developing search strategies.

Conflicts of Interest: The authors declare no conflict of interest.

\section{References}

1. Bray, F.; Ferlay, J.; Soerjomataram, I.; Siegel, R.L.; Torre, L.A.; Jemal, A. Global cancer statistics 2018: GLOBOCAN estimates of incidence and mortality worldwide for 36 cancers in 185 countries. CA Cancer J. Clin. 2018, 68, 394-424, Correction in CA Cancer J. Clin. 2020, 70, 313. [CrossRef]

2. Nguyen, P.L.; Alibhai, S.M.; Basaria, S.; D’Amico, A.V.; Kantoff, P.W.; Keating, N.L.; Penson, D.F.; Rosario, D.J.; Tombal, B.; Smith, M.R. Adverse effects of androgen deprivation therapy and strategies to mitigate them. Eur. Urol. 2015, 67, 825-836. [CrossRef] [PubMed]

3. Edmunds, K.; Tuffaha, H.; Galvão, D.A.; Scuffham, P.; Newton, R.U. Incidence of the adverse effects of androgen deprivation therapy for prostate cancer: A systematic literature review. Support. Care Cancer 2020, 28, 2079-2093. [CrossRef] [PubMed]

4. Gilbert, S.M.; Kuo, Y.F.; Shahinian, V.B. Prevalent and incident use of androgen deprivation therapy among men with prostate cancer in the United States. Urol. Oncol. 2011, 29, 647-653. [CrossRef]

5. Grossmann, M.; Hamilton, E.J.; Gilfillan, C.; Bolton, D.; Joon, D.L.; Zajac, J.D. Bone and metabolic health in patients with non-metastatic prostate cancer who are receiving androgen deprivation therapy. Med. J. Aust. 2011, 194, 301-306. [CrossRef] [PubMed]

6. Abdollah, F.; Sammon, J.D.; Reznor, G.; Sood, A.; Schmid, M.; Klett, D.E.; Sun, M.; Aizer, A.A.; Choueiri, T.K.; Hu, J.C. Medical androgen deprivation therapy and increased non-cancer mortality in non-metastatic prostate cancer patients aged $\geq 66$ years. Eur. J. Surg. Oncol. 2015, 41, 1529-1539. [CrossRef] [PubMed]

7. Bourke, L.; Smith, D.; Steed, L.; Hooper, R.; Carter, A.; Catto, J.; Albertsen, P.C.; Tombal, B.; Payne, H.A.; Rosario, D.J. Exercise for Men with Prostate Cancer: A Systematic Review and Meta-analysis. Eur. Urol. 2016, 69, 693-703. [CrossRef] [PubMed]

8. Gardner, J.R.; Livingston, P.M.; Fraser, S.F. Effects of exercise on treatment-related adverse effects for patients with prostate cancer receiving androgen-deprivation therapy: A systematic review. J. Clin. Oncol. 2014, 32, 335-346. [CrossRef] [PubMed]

9. Vashistha, V.; Singh, B.; Kaur, S.; Prokop, L.J.; Kaushik, D. The Effects of Exercise on Fatigue, Quality of Life, and Psychological Function for Men with Prostate Cancer: Systematic Review and Meta-analyses. Eur. Urol. Focus 2016, 2, 284-295. [CrossRef] [PubMed]

10. Lopez, P.; Taaffe, D.R.; Newton, R.U.; Buffart, L.M.; Galvão, D.A. What is the minimal dose for resistance exercise effectiveness in prostate cancer patients? Systematic review and meta-analysis on patient-reported outcomes. Prostate Cancer Prostatic Dis. 2021, 24, 465-481. [CrossRef] [PubMed] 
11. Bigaran, A.; Zopf, E.; Gardner, J.; Gerche, A.L.; Murphy, D.G.; Howden, E.J.; Baker, M.K.; Cormie, P. The effect of exercise training on cardiometabolic health in men with prostate cancer receiving androgen deprivation therapy: A systematic review and meta-analysis. Prostate Cancer Prostatic Dis. 2021, 24, 35-48. [CrossRef] [PubMed]

12. Zdravkovic, A.; Hasenöhrl, T.; Palma, S.; Crevenna, R. Effects of resistance exercise in prostate cancer patients: A systematic review update as of March 2020. Wien. Klin. Wochenschr. 2020, 132, 452-463. [CrossRef]

13. Kenfield, S.A.; Stampfer, M.J.; Giovannucci, E.; Chan, J.M. Physical activity and survival after prostate cancer diagnosis in the health professionals follow-up study. J. Clin. Oncol. 2011, 29, 726-732. [CrossRef] [PubMed]

14. Caspersen, C.J.; Powell, K.E.; Christenson, G.M. Physical activity, exercise, and physical fitness: Definitions and distinctions for health-related research. Public Health Rep. 1985, 100, 126-131. [PubMed]

15. Wang, Y.; Xu, D. Effects of aerobic exercise on lipids and lipoproteins. Lipids Health Dis. 2017, 16, 132. [CrossRef] [PubMed]

16. Knuttgen, H.; Kraemer, W.J. Terminology and measurement in exercise performance. Sport Sci. Res. 1987, 1, 1-10.

17. Higgins, J.P.T.; Savović, J.; Page, M.J.; Elbers, R.G.; Sterne, J.A.C. Chapter 8: Assessing risk of bias in a randomized trial. In Cochrane Handbook for Systematic Reviews of Interventions Version 6.2 (Updated February 2021); Higgins, J.P.T., Thomas, J., Chandler, J., Cumpston, M., Li, T., Page, M.J., Welch, V.A., Eds.; Cochrane: Chichester, UK, 2021.

18. McKenzie, J.E.; Brennan, S.E.; Ryan, R.E.; Thomson, H.J.; Johnston, R.V. Chapter 9: Summarizing study characteristics and preparing for synthesis. In Cochrane Handbook for Systematic Reviews of Interventions Version 6.2 (Updated February 2021); Higgins, J.P.T., Thomas, J., Chandler, J., Cumpston, M., Li, T., Page, M.J., Welch, V.A., Eds.; Cochrane: Chichester, UK, 2021.

19. Higgins, J.P.; Thompson, S.G.; Deeks, J.J.; Altman, D.G. Measuring inconsistency in meta-analyses. BMJ 2003, 327, 557-560. [CrossRef] [PubMed]

20. Egger, M.; Davey Smith, G.; Schneider, M.; Minder, C. Bias in meta-analysis detected by a simple, graphical test. BMJ 1997, 315, 629-634. [CrossRef] [PubMed]

21. Dalton, J.E.; Bolen, S.D.; Mascha, E.J. Publication Bias: The Elephant in the Review. Anesth. Analg. 2016, 123, 812-813. [CrossRef] [PubMed]

22. Bjerre, E.D.; Brasso, K.; Jørgensen, A.B.; Petersen, T.H.; Eriksen, A.R.; Tolver, A.; Christensen, J.F.; Poulsen, M.H.; Madsen, S.S.; Østergren, P.B. Football Compared with Usual Care in Men with Prostate Cancer (FC Prostate Community Trial): A Pragmatic Multicentre Randomized Controlled Trial. Sports Med. 2019, 49, 145-158. [CrossRef] [PubMed]

23. Bourke, L.; Gilbert, S.; Hooper, R.; Steed, L.A.; Joshi, M.; Catto, J.W.F.; Saxton, J.M.; Rosorio, D.J. Lifestyle changes for improving disease-specific quality of life in sedentary men on long-term androgen-deprivation therapy for advanced prostate cancer: A randomised controlled trial. Eur. Urol. 2014, 65, 865-872. [CrossRef] [PubMed]

24. Bourke, L.; Stevenson, R.; Turner, R.; Hooper, R.; Sasieni, P.; Greasley, R.; Morrissey, D.; Loosemore, M.; Fisher, A.; Payne, H.; et al. Exercise training as a novel primary treatment for localised prostate cancer: A multi-site randomised controlled phase II study. Sci. Rep. 2018, 8, 8374. [CrossRef] [PubMed]

25. Cormie, P.; Newton, R.U.; Spry, N.; Joseph, D.; Taaffe, D.R.; Galvão, D.A. Safety and efficacy of resistance exercise in prostate cancer patients with bone metastases [published correction appears in Prostate Cancer Prostatic Dis. 2015 Jun;18(2):196]. Prostate Cancer Prostatic Dis. 2013, 16, 328-335. [CrossRef] [PubMed]

26. Cormie, P.; Galvão, D.A.; Spry, N.; Joseph, D.; Chee, R.; Taaffe, D.R.; Chambers, S.K.; Newton, R.U. Can supervised exercise prevent treatment toxicity in patients with prostate cancer initiating androgen-deprivation therapy: A randomised controlled trial. BJU Int. 2015, 115, 256-266. [CrossRef] [PubMed]

27. Dawson, J.K.; Dorff, T.B.; Schroeder, E.T.; Lane, C.J.; Gross, M.E.; Dieli-Conwright, C.M. Impact of resistance training on body composition and metabolic syndrome variables during androgen deprivation therapy for prostate cancer: A pilot randomized controlled trial. BMC Cancer 2018, 18, 368. [CrossRef] [PubMed]

28. Dieperink, K.B.; Johansen, C.; Hansen, S.; Wagner, L.; Andersen, K.K.; Minet, L.R.; Hansen, O. The effects of multidisciplinary rehabilitation: RePCa-a randomised study among primary prostate cancer patients. Br. J. Cancer 2013, 109, 3005-3013. [CrossRef] [PubMed]

29. Eriksen, A.K.; Hansen, R.D.; Borre, M.; Larsen, R.G.; Jensen, J.M.; Overgaard, K.; Borre, M.; Kyrø, C.; Landberg, R.; Olsen, A.; et al. A lifestyle intervention among elderly men on active surveillance for non-aggressive prostate cancer: A randomised feasibility study with whole-grain rye and exercise. Trials 2017, 18, 20. [CrossRef]

30. Focht, B.C.; Lucas, A.R.; Grainger, E.; Simpson, C.; Fairman, C.M.; Thomas-Ahner, J.M.; Buell, J.; Monk, J.P.; Mortazavi, A.; Clinton, S.K. Effects of a Group-Mediated Exercise and Dietary Intervention in the Treatment of Prostate Cancer Patients Undergoing Androgen Deprivation Therapy: Results From the IDEA-P Trial. Ann. Behav. Med. 2018, 52, 412-428. [CrossRef] [PubMed]

31. Galvão, D.A.; Taaffe, D.R.; Spry, N.; Joseph, D.; Newton, R.U. Combined resistance and aerobic exercise program reverses muscle loss in men undergoing androgen suppression therapy for prostate cancer without bone metastases: A randomized controlled trial. J. Clin. Oncol. 2010, 28, 340-347. [CrossRef]

32. Galvão, D.A.; Spry, N.; Denham, J.; Taaffe, D.R.; Cormie, P.; Joseph, D.; Lamb, D.S.; Chambers, S.K.; Newton, R.U. A multicentre year-long randomised controlled trial of exercise training targeting physical functioning in men with prostate cancer previously treated with androgen suppression and radiation from TROG 03.04 RADAR. Eur. Urol. 2014, 65, 856-864. [CrossRef] [PubMed]

33. Galvão, D.A.; Taaffe, D.R.; Spry, N.; Cormie, P.; Joseph, D.; Chambers, S.K.; Chee, R.; Peddle-Mcintyre, C.J.; Hart, N.H.; Baumann, F.T.; et al. Exercise Preserves Physical Function in Prostate Cancer Patients with Bone Metastases. Med. Sci. Sports Exerc. 2018, 50, 393-399. [CrossRef] 
34. Hébert, J.R.; Hurley, T.G.; Harmon, B.E.; Heiney, S.; Hebert, C.J.; Steck, S.E. A diet, physical activity, and stress reduction intervention in men with rising prostate-specific antigen after treatment for prostate cancer. Cancer Epidemiol. 2012, 36, e128-e136. [CrossRef] [PubMed]

35. Hojan, K.; Kwiatkowska-Borowczyk, E.; Leporowska, E.; Górecki, M.; Ozga-Majchrzak, O.; Milecki, T.; Milecki, P. Physical exercise for functional capacity, blood immune function, fatigue, and quality of life in high-risk prostate cancer patients during radiotherapy: A prospective, randomized clinical study. Eur. J. Phys. Rehabil. Med. 2016, 52, 489-501. [PubMed]

36. Hojan, K.; Kwiatkowska-Borowczyk, E.; Leporowska, E.; Milecki, P. Inflammation, cardiometabolic markers, and functional changes in men with prostate cancer. A randomized controlled trial of a 12-month exercise program. Pol. Arch. Intern. Med. 2017, 127, 25-35. [CrossRef] [PubMed]

37. Hvid, T.; Winding, K.; Rinnov, A.; Dejgaard, T.; Thomsen, C.; Iversen, P.; Brasso, K.; Mikines, K.J.; van Hall, G.; Lindegaard, B.; et al. Endurance training improves insulin sensitivity and body composition in prostate cancer patients treated with androgen deprivation therapy. Endocr. Relat. Cancer 2013, 20, 621-632. [CrossRef] [PubMed]

38. Jones, L.W.; Hornsby, W.E.; Freedland, S.J.; Lane, A.; West, M.J.; Moul, J.W.; Ferrandino, M.N.; Allen, J.D.; Kenjale, A.A.; Thomas, S.M.; et al. Effects of nonlinear aerobic training on erectile dysfunction and cardiovascular function following radical prostatectomy for clinically localized prostate cancer. Eur. Urol. 2014, 65, 852-855. [CrossRef] [PubMed]

39. Livingston, P.M.; Craike, M.J.; Salmon, J.; Courneya, K.S.; Gaskin, C.J.; Fraser, S.F.; Mohebbi, M.; Broadbent, S.; Botti, M.; Kent, B.; et al. Effects of a clinician referral and exercise program for men who have completed active treatment for prostate cancer: A multicenter cluster randomized controlled trial (ENGAGE). Cancer 2015, 121, 2646-2654. [CrossRef]

40. Monga, U.; Garber, S.L.; Thornby, J.; Vallbona, C.; Kerrigan, A.J.; Monga, T.N.; Zimmermann, K.P. Exercise prevents fatigue and improves quality of life in prostate cancer patients undergoing radiotherapy. Arch. Phys. Med. Rehabil. 2007, 88, 1416-1422. [CrossRef]

41. Ndjavera, W.; Orange, S.T.; O’Doherty, A.F.; Leicht, A.S.; Rochester, M.; Mills, R.; Saxton, J. Exercise-induced attenuation of treatment side-effects in patients with newly diagnosed prostate cancer beginning androgen-deprivation therapy: A randomised controlled trial. BJU Int. 2020, 125, 28-37. [CrossRef] [PubMed]

42. Nilsen, T.S.; Raastad, T.; Skovlund, E.; Courneya, K.S.; Langberg, C.W.; Lilleby, W.; Fosså, S.D.; Thorsen, L. Effects of strength training on body composition, physical functioning, and quality of life in prostate cancer patients during androgen deprivation therapy. Acta Oncol. 2015, 54, 1805-1813. [CrossRef] [PubMed]

43. O'Neill, R.F.; Haseen, F.; Murray, L.J.; O'Sullivan, J.M.; Cantwell, M.M. A randomised controlled trial to evaluate the efficacy of a 6-month dietary and physical activity intervention for patients receiving androgen deprivation therapy for prostate cancer. J. Cancer Surviv. 2015, 9, 431-440. [CrossRef] [PubMed]

44. Park, S.W.; Kim, T.N.; Nam, J.K.; Ha, H.K.; Shin, D.G.; Lee, W.; Kim, M.-S.; Chung, M.K. Recovery of overall exercise ability, quality of life, and continence after 12-week combined exercise intervention in elderly patients who underwent radical prostatectomy: A randomized controlled study. Urology 2012, 80, 299-305. [CrossRef] [PubMed]

45. Segal, R.J.; Reid, R.D.; Courneya, K.S.; Malone, S.C.; Parliament, M.B.; Scott, C.G.; Venner, P.M.; Quinney, H.A.; Jones, L.W.; Lovinec, M.E.; et al. Resistance exercise in men receiving androgen deprivation therapy for prostate cancer. J. Clin. Oncol. 2003, 21, 1653-1659. [CrossRef] [PubMed]

46. Segal, R.J.; Reid, R.D.; Courneya, K.S.; Sigal, R.J.; Kenny, G.P.; Prud' Homme, D.G.; Malone, S.C.; Wells, G.A.; Scott, C.G.; D'Angelo, M.E.S. Randomized controlled trial of resistance or aerobic exercise in men receiving radiation therapy for prostate cancer. J. Clin. Oncol. 2009, 27, 344-351. [CrossRef] [PubMed]

47. Taaffe, D.R.; Newton, R.U.; Spry, N.; Joseph, D.; Chambers, S.K.; Gardiner, R.A.; Wall, B.A.; Cormie, P.; Bolam, K.A.; Galvāo, D.A. Effects of Different Exercise Modalities on Fatigue in Prostate Cancer Patients Undergoing Androgen Deprivation Therapy: A Year-long Randomised Controlled Trial. Eur. Urol. 2017, 72, 293-299. [CrossRef] [PubMed]

48. Taaffe, D.R.; Galvão, D.A.; Spry, N.; Joseph, D.; Chambers, S.K.; Gardiner, R.A.; Hayne, D.; Cormie, P.; Shum, D.H.K.; Newton, R.U. Immediate versus delayed exercise in men initiating androgen deprivation: Effects on bone density and soft tissue composition. BJU Int. 2019, 123, 261-269. [CrossRef] [PubMed]

49. Uth, J.; Hornstrup, T.; Schmidt, J.F.; Christensen, J.F.; Frandsen, C.; Christensen, K.B.; Helge, E.W.; Brasso, K.; Rørth, M.; Midtgaard, J.; et al. Football training improves lean body mass in men with prostate cancer undergoing androgen deprivation therapy. Scand. J. Med. Sci. Sports 2014, 24 (Suppl. 1), 105-112. [CrossRef] [PubMed]

50. Windsor, P.M.; Nicol, K.F.; Potter, J. A randomized, controlled trial of aerobic exercise for treatment-related fatigue in men receiving radical external beam radiotherapy for localized prostate carcinoma. Cancer 2004, 101, 550-557. [CrossRef] [PubMed]

51. Wall, B.A.; Galvão, D.A.; Fatehee, N.; Taaffe, D.R.; Spry, N.; Joseph, D.; Hebert, J.J.; Newton, R.U. Exercise Improves V O2max and Body Composition in Androgen Deprivation Therapy-treated Prostate Cancer Patients. Med. Sci. Sports Exerc. 2017, 49, 1503-1510. [CrossRef] [PubMed]

52. Winters-Stone, K.M.; Dobek, J.C.; Bennett, J.A.; Maddalozzo, G.F.; Ryan, C.W.; Beer, T.M. Skeletal response to resistance and impact training in prostate cancer survivors. Med. Sci. Sports Exerc. 2014, 46, 1482-1488. [CrossRef] [PubMed]

53. Winters-Stone, K.M.; Lyons, K.S.; Dobek, J.; Dieckmann, N.F.; Bennett, J.A.; Nail, L.; Beer, T.M. Benefits of partnered strength training for prostate cancer survivors and spouses: Results from a randomized controlled trial of the Exercising Together project. J. Cancer Surviv. 2016, 10, 633-644. [CrossRef] [PubMed] 
54. Mardani, A.; Pedram Razi, S.; Mazaheri, R.; Haghani, S.; Vaismoradi, M. Effect of the exercise programme on the quality of life of prostate cancer survivors: A randomized controlled trial. Int. J. Nurs. Pract. 2021, 27, e12883. [CrossRef] [PubMed]

55. Alberga, A.S.; Segal, R.J.; Reid, R.D.; Scott, C.G.; Sigal, R.G.; Khandwala, F.; Jeffey, J.; Wells, G.A.; Kenny, G.P. Age and androgendeprivation therapy on exercise outcomes in men with prostate cancer. Support. Care Cancer 2012, 20, 971-981. [CrossRef] [PubMed]

56. Bourke, L.; Doll, H.; Crank, H.; Daley, A.; Rosario, D.; Saxton, J.M. Lifestyle intervention in men with advanced prostate cancer receiving androgen suppression therapy: A feasibility study. Cancer Epidemiol. Biomark. Prev. 2011, 20, 647-657. [CrossRef] [PubMed]

57. Kapur, G.; Windsor, P.M.; McCowan, C. The effect of aerobic exercise on treatment-related acute toxicity in men receiving radical external beam radiotherapy for localised prostate cancer. Eur. J. Cancer Care Engl. 2010, 19, 643-647. [CrossRef] [PubMed]

58. Gilbert, S.E.; Tew, G.A.; Fairhurst, C.; Bourke, L.; Saxton, J.M.; Winter, E.M.; Rosario, D.J. Effects of a lifestyle intervention on endothelial function in men on long-term androgen deprivation therapy for prostate cancer. Br. J. Cancer 2016, 114, 401-408. [CrossRef] [PubMed]

59. Galvão, D.A.; Taaffe, D.R.; Spry, N.; Joseph, D.; Newton, R.U. Acute versus chronic exposure to androgen suppression for prostate cancer: Impact on the exercise response. J. Urol. 2011, 186, 1291-1297. [CrossRef]

60. Cormie, P.; Newton, R.U.; Taaffe, D.R.; Spry, N.; Joseph, D.; Hamid, M.A.; Galvão, D.A. Exercise maintains sexual activity in men undergoing androgen suppression for prostate cancer: A randomized controlled trial. Prostate Cancer Prostatic Dis. 2013, 16, 170-175. [CrossRef] [PubMed]

61. Buffart, L.M.; Kalter, J.; Sweegers, M.G.; Courneya, K.S.; Newton, R.U.; Aaronson, N.K.; Jacobsen, P.B.; May, A.M.; Galvāo, D.A.; Chinapaw, M.J.; et al. Effects and moderators of exercise on quality of life and physical function in patients with cancer: An individual patient data meta-analysis of 34 RCTs. Cancer Treat. Rev. 2017, 52, 91-104. [CrossRef]

62. Taaffe, D.R.; Buffart, L.M.; Newton, R.U.; Spry, N.; Denham, J.; Joseph, D.; Lamb, D.; Chambers, S.K.; Galvao, D.A. Time on androgen deprivation therapy and adaptations to exercise: Secondary analysis from a 12-month randomized controlled trial in men with prostate cancer. BJU Int. 2018, 121, 194-202. [CrossRef]

63. Courneya, K.S.; Segal, R.J.; Reid, R.D.; Jones, L.W.; Malone, S.C.; Venner, P.M.; Parliament, M.B.; Scott, C.G.; Quinney, H.A.; Wells, G.A. Three independent factors predicted adherence in a randomized controlled trial of resistance exercise training among prostate cancer survivors. J. Clin. Epidemiol. 2004, 57, 571-579. [CrossRef] [PubMed]

64. Gaskin, C.J.; Fraser, S.F.; Owen, P.J.; Craike, M.; Orellana, L.; Livingston, P.M. Fitness outcomes from a randomised controlled trial of exercise training for men with prostate cancer: The ENGAGE study. J. Cancer Surviv. 2016, 10, 972-980. [CrossRef] [PubMed]

65. Gaskin, C.J.; Craike, M.; Mohebbi, M.; Courneya, K.S.; Livingston, P.M. A Clinician Referral and 12-Week Exercise Training Program for Men with Prostate Cancer: Outcomes to 12 Months of the ENGAGE Cluster Randomized Controlled Trial. J. Phys. Act. Health 2017, 14, 353-359. [CrossRef] [PubMed]

66. Newton, R.U.; Galvão, D.A.; Spry, N.; Joseph, D.; Chambers, S.K.; Gardiner, R.A.; Wall, B.A.; Bolam, K.A.; Taaffe, D.R. Exercise Mode Specificity for Preserving Spine and Hip Bone Mineral Density in Prostate Cancer Patients. Med. Sci. Sports Exerc. 2019, 51, 607-614. [CrossRef] [PubMed]

67. Buffart, L.M.; Newton, R.U.; Chinapaw, M.J.; Taaffe, D.R.; Spry, N.A.; Denham, J.W.; Joseph, D.J.; Lamb, D.S.; Brug, J.; Galvão, D.A. The effect, moderators, and mediators of resistance and aerobic exercise on health-related quality of life in older long-term survivors of prostate cancer. Cancer 2015, 121, 3367-3368. [CrossRef]

68. Nilsen, T.S.; Thorsen, L.; Kirkegaard, C.; Ugelstad, I.; Fosså, S.D.; Raastad, T. The effect of strength training on muscle cellular stress in prostate cancer patients on ADT. Endocr. Connect. 2016, 5, 74-82. [CrossRef]

69. Uth, J.; Hornstrup, T.; Christensen, J.F.; Christensen, K.B.; Jørgensen, N.R.; Brasso, K.; Jakobsen, M.D.; Sundstrup, E.; Anderson, L.L.; Rørth, M.; et al. Efficacy of recreational football on bone health, body composition, and physical functioning in men with prostate cancer undergoing androgen deprivation therapy: 32-week follow-up of the FC prostate randomised controlled trial. Osteoporos. Int. 2016, 27, 1507-1518. [CrossRef]

70. Uth, J.; Fristrup, B.; Haahr, R.D.; Haahr, R.D.; Brasso, K.; Helge, J.W.; Rørth, M.; Midtgaard, J.; Helge, E.W.; Krustrup, P.; et al. Football training over 5 years is associated with preserved femoral bone mineral density in men with prostate cancer. Scand. J. Med. Sci. Sports 2018, 28 (Suppl. 1), 61-73. [CrossRef] [PubMed]

71. Winters-Stone, K.M.; Dobek, J.C.; Bennett, J.A.; Dieckmann, N.F.; Maddalozzo, G.F.; Ryan, C.W.; Beer, T.M. Resistance training reduces disability in prostate cancer survivors on androgen deprivation therapy: Evidence from a randomized controlled trial. Arch. Phys. Med. Rehabil. 2015, 96, 7-14. [CrossRef] [PubMed]

72. Newton, R.U.; Galvão, D.A.; Spry, N.; Joseph, D.; Chambers, S.K.; Gardiner, R.A.; Hayne, D.; Taaffe, D.R. Timing of exercise for muscle strength and physical function in men initiating ADT for prostate cancer. Prostate Cancer Prostatic Dis. 2020, 23, 457-464. [CrossRef]

73. Dieperink, K.B.; Hansen, S.; Wagner, L.; Minet, L.R.; Hansen, O. Long-term follow-up 3 years after a randomized rehabilitation study among radiated prostate cancer survivors. J. Cancer Surviv. 2021, 15, 668-676. [CrossRef] [PubMed]

74. Bjerre, E.D.; Weller, S.; Poulsen, M.H.; Poulsen, M.H.; Madsen, S.S.; Bjerre, R.D.; Østergren, P.B.; Borre, M.; Brasso, K.; Midtgaard, J. Safety and Effects of Football in Skeletal Metastatic Prostate Cancer: A Subgroup Analysis of the FC Prostate Community Randomised Controlled Trial. Sports Med. -Open 2021, 7, 27. [CrossRef] [PubMed] 
75. Schumacher, O.; Galvão, D.A.; Taaffe, D.R.; Spry, N.; Joseph, D.; Tang, C.; Chee, R.; Newton, R.U. Effect of Exercise Adjunct to Radiation and Androgen Deprivation Therapy on Patient-Reported Treatment Toxicity in Men With Prostate Cancer: A Secondary Analysis of 2 Randomized Controlled Trials. Pract. Radiat. Oncol. 2021, 11, 215-225. [CrossRef] [PubMed]

76. Galvão, D.A.; Newton, R.U.; Chambers, S.K.; Spry, N.; Joseph, D.; Gardiner, R.A.; Fairman, C.M.; Taaffe, D.R. Psychological distress in men with prostate cancer undertaking androgen deprivation therapy: Modifying effects of exercise from a year-long randomized controlled trial. Prostate Cancer Prostatic Dis. 2021, 24, 758-766. [CrossRef]

77. Newton, R.U.; Mavropalias, G.; Fragala, M.S.; Kraemer, W.J.; Häkkkinen, K.; Taaffe, D.R.; Spry, N.; Joseph, D.; Galvāo, D.A. Radiotherapy before or during androgen-deprivation therapy does not blunt the exercise-induced body composition protective effects in prostate cancer patients: A secondary analysis of two randomized controlled trials. Exp. Gerontol. 2021, 151, 111427. [CrossRef] [PubMed]

78. Mina, D.S.; Alibhai, S.M.H.; Matthew, A.G.; Guglietti, C.L.; Pirbaglou, M.; Trachtenberg, J.; Ritvo, P. A randomized trial of aerobic versus resistance exercise in prostate cancer survivors. J. Aging Phys. Act. 2013, 21, 455-478. [CrossRef] [PubMed]

79. Kelley, G.A.; Kelley, K.S. Exercise and cancer-related fatigue in adults: A systematic review of previous systematic reviews with meta-analyses. BMC Cancer 2017, 17, 693. [CrossRef] [PubMed]

80. Charalambous, A.; Kouta, C. Cancer Related Fatigue and Quality of Life in Patients with Advanced Prostate Cancer Undergoing Chemotherapy. Biomed. Res. Int. 2016, 2016, 3989286. [CrossRef]

81. McCabe, R.M.; Grutsch, J.F.; Braun, D.P.; Nutakki, S.B. Fatigue as a Driver of Overall Quality of Life in Cancer Patients. PLoS ONE 2015, 10, e0130023. [CrossRef]

82. Buffart, L.M.; Sweegers, M.G.; May, A.M.; Chinapaw, M.J.; van Vulpen, J.K.; Newton, R.U.; Galvão, D.A.; Aaronson, N.K.; Stuiver, M.M.; Jacobsen, P.B.; et al. Targeting Exercise Interventions to Patients with Cancer in Need: An Individual Patient Data Meta-Analysis. J. Natl. Cancer Inst. 2018, 110, 1190-1200. [CrossRef]

83. Wedell-Neergaard, A.S.; Krogh-Madsen, R.; Petersen, G.L.; Hansen, Å.M.; Pedersen, B.K.; Lund, R.; Bruunsgaard, H. Cardiorespiratory fitness and the metabolic syndrome: Roles of inflammation and abdominal obesity. PLoS ONE 2018, 13, e0194991. [CrossRef] [PubMed]

84. Schmidt, K.; Vogt, L.; Thiel, C.; Jäger, E.; Banzer, W. Validity of the six-minute walk test in cancer patients. Int. J. Sports Med. 2013, 34, 631-636. [CrossRef] [PubMed]

85. Hayes, S.C.; Newton, R.U.; Spence, R.R.; Galvão, D.A. The Exercise and Sports Science Australia position statement: Exercise medicine in cancer management. J. Sci. Med. Sport 2019, 22, 1175-1199. [CrossRef] [PubMed]

86. National Institute for Health and Care Excellence (2019). Prostate Cancer: Diagnosis and Management (NICE Guideline 131) Available online: https:/ / www.nice.org.uk/guidance/ng131/chapter/recommendations\#terms-used-in-this-guideline (accessed on 1 August 2021).

87. Wayne, P.M.; Lee, M.S.; Novakowski, J.; Osypiuk, K.; Ligibel, J.; Carlson, L.E.; Song, R. Tai Chi and Qigong for cancer-related symptoms and quality of life: A systematic review and meta-analysis. J. Cancer Surviv. 2018, 12, 256-267. [CrossRef] [PubMed]

88. Ben-Josef, A.M.; Chen, J.; Wileyto, P.; Doucette, A.; Bakelman, J.; Christodouleas, J.; Deville, C.; Vapiwala, N. Effect of Eischens Yoga During Radiation Therapy on Prostate Cancer Patient Symptoms and Quality of Life: A Randomized Phase II Trial. Int. J. Radiat. Oncol. Biol. Phys. 2017, 98, 1036-1044. [CrossRef] [PubMed]

89. Neil-Sztramko, S.E.; Medysky, M.E.; Campbell, K.L.; Bland, K.A.; Winters-Stone, K.M. Attention to the principles of exercise training in exercise studies on prostate cancer survivors: A systematic review. BMC Cancer 2019, 19, 321. [CrossRef] 